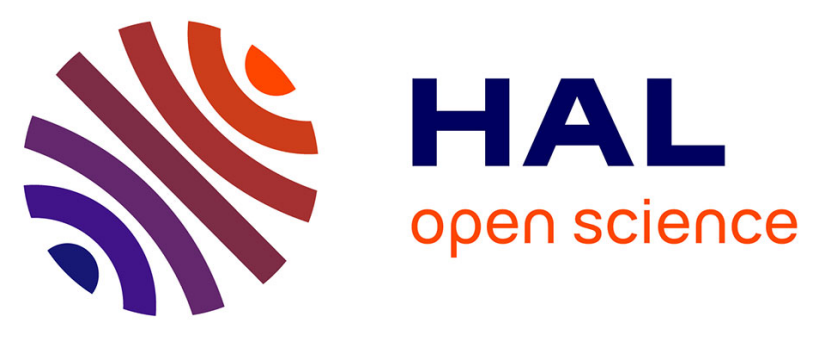

\title{
Evidence of inter-particles collision effect in airflow resuspension of poly-dispersed tungsten particles in monolayer deposits
}

\author{
Anthony Rondeau, Samuel Peillon, Ana Maria Vidalès, Jesica Benito, Rodolfo \\ Unac, Francois Gensdarmes, Jean-Christophe Sabroux
}

\section{To cite this version:}

Anthony Rondeau, Samuel Peillon, Ana Maria Vidalès, Jesica Benito, Rodolfo Unac, et al.. Evidence of inter-particles collision effect in airflow resuspension of poly-dispersed tungsten particles in monolayer deposits. Journal of Aerosol Science, 2021, 154, pp.105735. 10.1016/j.jaerosci.2020.105735 . hal03149291

\section{HAL Id: hal-03149291 \\ https://hal.science/hal-03149291}

Submitted on 22 Feb 2021

HAL is a multi-disciplinary open access archive for the deposit and dissemination of scientific research documents, whether they are published or not. The documents may come from teaching and research institutions in France or abroad, or from public or private research centers.
L'archive ouverte pluridisciplinaire HAL, est destinée au dépôt et à la diffusion de documents scientifiques de niveau recherche, publiés ou non, émanant des établissements d'enseignement et de recherche français ou étrangers, des laboratoires publics ou privés.

\section{(ㅇ)(1) $\$$}

Distributed under a Creative Commons Attribution - NonCommercial - NoDerivatives 44.0 


\title{
Evidence of inter-particles collision effect in airflow resuspension of poly-dispersed non-spherical tungsten particles in monolayer deposits
}

\author{
Anthony Rondeau ${ }^{1}$, Samuel Peillon ${ }^{1}$, Ana M. Vidales ${ }^{2}$, Jesica Benito ${ }^{2}$, Rodolfo Uñac ${ }^{2}$, Jean- \\ Christophe Sabroux ${ }^{1}$, François Gensdarmes ${ }^{1}$ \\ ${ }^{1}$ Institut de Radioprotection et de Sûreté Nucléaire (IRSN), PSN-RES, SCA, Gif-sur-Yvette, 91192, France. \\ ${ }^{2}$ INFAP, CONICET, Universidad Nacional de San Luis, San Luis, D5700HHW, Argentina.
}

\begin{abstract}
Poly-dispersed particle resuspension by turbulent airflow was experimentally studied in a wind tunnel called BISE. The set-up was carefully validated to produce reference airflows with three absolute pressures and friction velocities values. Monolayer deposits of poly-dispersed tungsten particles on tungsten surfaces were used for the experiments. The fraction of detached particles by size bin from $1 \mu \mathrm{m}$ to $50 \mu \mathrm{m}$ was quantified to analyze the effects of friction velocity, absolute pressure and particles concentration on surface. Comparison of the results with Rock'n Roll and Monte-Carlo resuspension modeling revealed discrepancies for particles behavior with diameter below $4.5 \mu \mathrm{m}$. These discrepancies could not be understood by assessment of particle-surface adhesion force distribution carried out with Atomic Force Microscopy analysis. For particle surface concentrations ranging from 35 to $100 \mathrm{~mm}^{-2}$, a collision effect was identified due to the concentration and poly-dispersion of particles deposited on the surface. Indeed, when analyzing the experimental data, a good linear correlation is obtained between the fraction of the small particles detached and a collision parameter, demonstrating that collisions could be responsible for the unexpectedly high resuspended fraction observed for particle size below $4.5 \mu \mathrm{m}$ in diameter. The experimental data and the analysis presented in the paper show that particle surface concentration and collision effects are clearly relevant and should be considered in the above-mentioned resuspension models, especially for poly-dispersed particles assessment.
\end{abstract}

Keywords: Resuspension, adhesion, particle, airflow, collision

\section{Introduction}

Apart from being an industrial problem, the dust resuspension (particles of $100 \mu \mathrm{m}$ diameter or less) is a natural phenomenon in many scenarios. For example, the particle resuspension is observed during volcanic eruptions, snow avalanches or sand storm, and is of a wide scientific and practical interest (Henry and Minier, 2014a; Boor et al., 2013; Gradon, 2009; Ziskind, 2006; Reeks and Hall, 2001). In the present work, we studied the aeraulic resuspension of particles, deposited on a flat surface, by a turbulent airflow in the framework of nuclear safety analysis. This phenomenon is mostly studied at atmospheric pressure. Nevertheless, development of fusion technology using tokamak type vacuum chamber requires assessing dust behaviour in low pressure airflow (Gélain et al., 2015) in case of a breach leading to a so called Loss of Vacuum Accident (LOVA). A vacuum chamber, like a particle accelerator or a tokamak, is usually linked to an ultra-clean environment, i.e., without dust. Indeed, if the plasma required for nuclear fusion reactions is not perfectly controlled in tokamaks, erosion of the plasma facing components in vacuum chamber results, leading thus to dust production (Roth et al., 2009). Thus, knowledge on particle re-suspension at low pressure in such industrial environment is nowadays mandatory and few data is available in the literature. For example, Rondeau et al., (2015a) obtained preliminary results on resuspension of multilayer deposits of particles $10-30 \mu \mathrm{m}$ in diameter at different pressure using a facility (The wind tunnel AWTS-II of the Aarhus University) initially dedicated to planetology research. 
The natural phenomenon of dust resuspension at low pressure is observed during storms in the Martian atmosphere, where the ground level pressure is about $7 \mathrm{mbar}$. In the vacuum of what is called the lunar atmosphere (from $10^{-8}$ to $10^{-10} \mathrm{~Pa}$ ), the dust is essentially resuspended by the action of electric forces due to the photoelectric effect (solar UV $<100 \mathrm{~nm}$ ) and the solar wind. Consequently, most data on the effect of low pressure on the particle resuspension mechanism, with or without electric forces, stem from planetology studies (Balme and Hagermann, 2006; Renno and Kok, 2008; Kok et al., 2012).

The concentration of the particles on the surface is decisive for collision occurrence in wind tunnel experiments. For example, Ibrahim et al. (2004) reported collisions for mono-dispersed particles of $70 \mu \mathrm{m}$, with number concentrations of 0.5 and $3 \mathrm{~mm}^{-2}$. They found that collisions increase particle removal and play a significant role at high particle concentration. On the other hand, the effect of the poly-dispersion of the particles in the above scenarios could make predictions still more complex.

The lack of experiments with poly-dispersed particles, at moderate or high concentrations and low pressure, stimulated us to perform the present study to provide new results which could be relevant not only for nuclear accidents applications and planetology but also important for solar cells pollution (Moutinho et al., 2017), atmosphere contamination nearby the opencast mining (Csavina et al. 2012; Stovern et al. 2014; Bisquert, Peñas-Castejón and García-Fernández 2017) and surface cleaning for micro and nanoelectronic technology.

In the first part of this study, we present experimental data on particle resuspension by turbulent airflow at atmospheric pressure and at reduced pressure, in a limited number of configurations but with careful validation of wind tunnel aeraulic and particle-surface adhesion forces. We highlight the fact that in the present experiments, the measurement of the roughness, adhesion force distribution and flow properties has been performed along with the resuspended particles fraction. In this way, just a few parameters could be left undetermined or used as possible tuning parameters for modeling the results. We focus our interest on poly-dispersed tungsten particle deposits following the dust collection in an actual tokamak, as achieved by Rondeau et al. (2015b) who measured tungsten particle diameter sizes between 0.1 and $30 \mu \mathrm{m}$.

The second part of the paper presents a comparison of the results with Rock'n Roll - RnR (Reeks and Hall, 2001) and Monte-Carlo (Benito et al., 2016) resuspension models. The comparison reveals some discrepancy for particles behaviour with diameter below $4.5 \mu \mathrm{m}$ which cannot be understood by the assessment of particle-surface adhesion force distribution. By a thorough analysis of the experimental data, the third part of the study puts into evidence a collision effect due to the concentration and the poly-dispersion of particles deposited on the surface which are not taken into account in the abovementioned resuspension models, as particles are considered independently.

\section{The BISE-II wind-tunnel}

In order to study the particle resuspension mechanism at low pressure, we used the BISE-II wind tunnel, located at the Saclay IRSN research centre. The BISE-II wind tunnel allows studying the resuspension mechanism of particles deposited on a surface at pressures fairly lower than atmospheric pressure. The BISE-II is the upgraded version of the BISE-I facility which was dedicated to study particle resuspension from a pile of powder at atmospheric pressure (Alloul-Marmor, 2002, Peillon et al., 2014, Zhang et al., 2017). Here, this new facility is described with its associated metrological devices, allowing us to measure the airflow parameters influencing the resuspension mechanism, namely, the friction velocity $u_{*}$, the surrounding pressure $P$ and the airflow forces fluctuation through the relative standard deviation of airflow velocity $f_{\text {rms }}$. Figure 1 presents the schematic diagram of the BISE-II facility, the total length of which extends to five metres. 


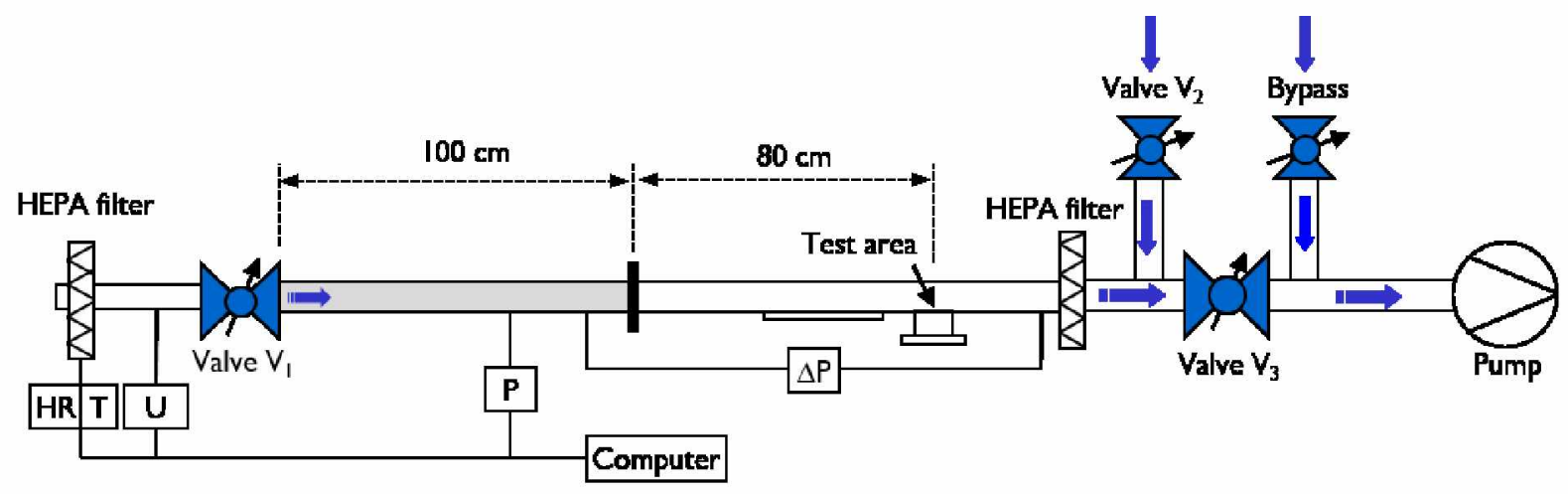

Figure 1 - Schematic diagram of the BISE-II facility.

In the BISE-II wind tunnel, the airflow is generated by means of a vacuum pump (Rietschle SMV 500) located downstream from the tunnel and having a steady volume flow rate of $560 \mathrm{~m}^{3} \cdot \mathrm{h}^{-1} \mathrm{STP}$, when the pump is powered by a $60 \mathrm{~Hz}$ mains. Valves $V_{1}$ and $V_{2}$ are used to set the airflow in terms of flow rate (hence, velocity) and absolute pressure. Valve $V_{3}$ allows switching the airflow from the test section to the bypass valve for inserting or extracting a sample from the wind tunnel without powering off the pump. Thanks to the bypass circuit, we can safeguard the valves $V_{1}$ and $V_{2}$ settings for the airflow velocity and the absolute pressure as selected for the test.

The free-stream velocities range from 0.5 to $40 \mathrm{~m} . \mathrm{s}^{-1}$ at pressures from 200 to $1,000 \mathrm{mbar}$ absolute. Two levels of velocities and pressures are set independently for the experiments performed in this study and are reported in Table 1 . The airflow velocity is measured by a thermal anemometer (TSI-8455 - indicated $\mathrm{U}$ on Figure 1) permanently set at atmospheric pressure, upstream the valve $\mathrm{V}_{1}$. The absolute pressure is measured between valve $\mathrm{V}_{1}$ and the test area. The temperature and humidity of the inlet airflow are measured by a sensor (Kimo Instruments TH300-PNB) located inside the HEPA filter housing. The test section of the facility is a rectangular duct with a length of $200 \mathrm{~cm}$, a width of $7.5 \mathrm{~cm}$ and a height of 6 $\mathrm{cm}$. This rectangular pipe is split into two parts: one part is made of stainless steel while the other is in Plexiglas for the upper wall, glass for the side walls (convenient for optical observations or measurements) and stainless steel for the bottom part which is grounded (white duct extending the grey duct in figure 1). For each experiment presented in this paper, the following parameters were continuously measured during the tests and will be indicated accordingly: absolute pressure $(P)$, airflow velocity $(U)$, friction velocity $\left(u_{*}\right)$, relative humidity $(R H)$ and temperature $(T)$.

\section{Airflow characterization}

\subsection{Determination of velocity profiles and turbulence intensities}

As mentioned above, during resuspension experiments, the airflow velocity is controlled with the TSI8455 anemometer located upstream the valve $\mathrm{V}_{1}$. A careful characterization of the different free-stream velocity profiles and turbulence intensities obtained in the test section was realized according to three experimental set velocities managed with the upstream anemometer.

Velocity vertical profile as well as turbulence intensity have been investigated using high-frequency hot-wire anemometry (Dantec Dynamics) and are depicted in Figure 2-(a) and 2-(b), respectively. These measurements were performed at atmospheric pressure over the stainless steel surface of the wind tunnel. The wind tunnel surface is smooth with roughness $R a<0.1 \mu \mathrm{m}$. Profiles are measured at the centre of the wind tunnel at the sample location $(y=3.75 \mathrm{~cm})$ with $\mathrm{z}$ the vertical position of the probe. The velocity profiles depicted in Figure 2 -(a) represent the mean velocity $\bar{u}$, measured by the highfrequency hot-wire anemometry, normalized by the maximum airflow velocity $U_{\max }$ measured at the centre $(z=3 \mathrm{~cm})$ of the duct. One can see that for the three free-stream velocities studied $(5,15$ and $\left.30 \mathrm{~m} . \mathrm{s}^{-1}\right)$, the profiles are established and characteristic of a turbulent flow. The airflow velocity near the wall (at $\mathrm{z}=1 \mathrm{~mm})$ is between $35 \%$ and $55 \%$ lower than the free-stream velocity at the centre of the 
tunnel. The airflow velocity profile at $5 \mathrm{~m} \cdot \mathrm{s}^{-1}$ is more spread out, the velocity at $\mathrm{z}=1 \mathrm{~mm}$ being 2.25 $\mathrm{m} \cdot \mathrm{s}^{-1}(\approx 45 \%$ of the free-stream velocity at the centre of the duct). In Figure 2-(b), the turbulence intensity, defined as the ratio of the standard deviation of the instantaneous velocity over the mean velocity $\bar{u}$, is presented. One should notice that measurements performed on the instantaneous velocity exhibit classical Gaussian distributions even for the closest location at $1 \mathrm{~mm}$ from the wall. It is found that the intensity of turbulence reaches $30 \%$ near the surface when the free-stream velocity is $5 \mathrm{~m} . \mathrm{s}^{-1}$ and is approximately $5 \%$ in the free flow zone. The turbulence profiles are much flatter for higher velocities, especially at $30 \mathrm{~m} \cdot \mathrm{s}^{-1}$, where the turbulence intensity goes from $10 \%$ near the surface to less than $5 \%$ in the free flow zone.
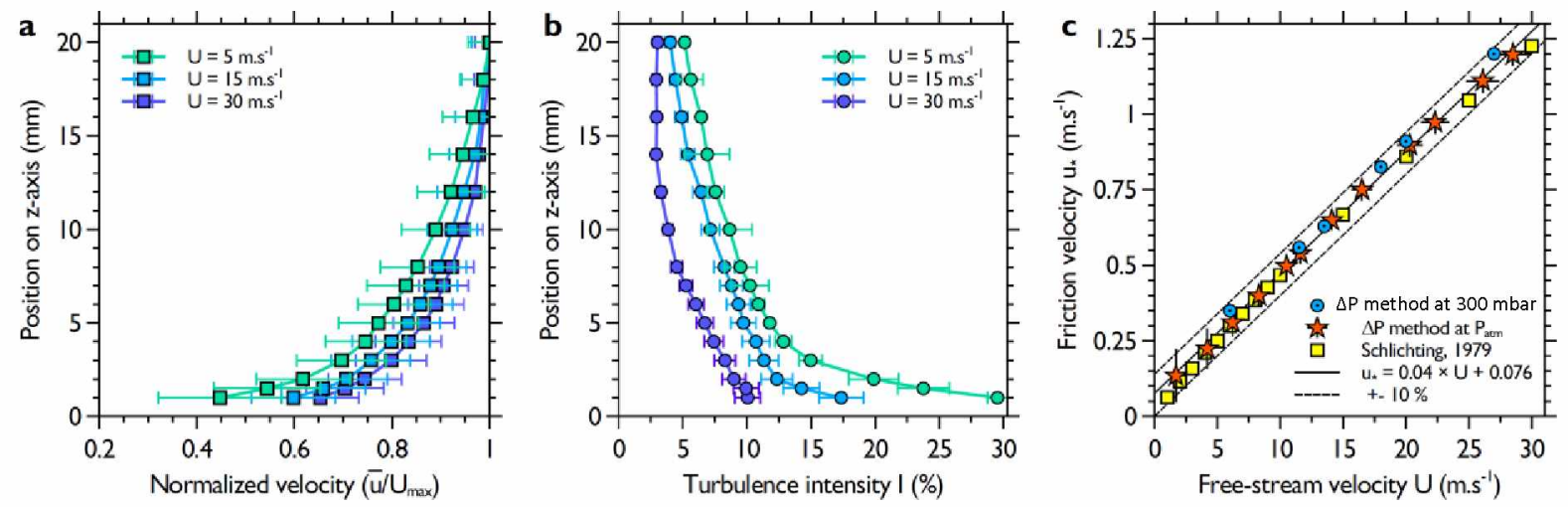

Figure 2 - Normalized velocity profiles (a) and turbulence intensity profiles (b) close to the surface at the centre of the deposit zone for $U=5,15$ and $30 \mathrm{~m} \cdot \mathrm{s}^{-1}$. (c) Friction velocity measured with the pressure drop method versus free-stream velocity.

As indicated by Biasi et al. (2001) for steady-state flows, the fluctuations of the airflow forces follow a Gaussian law and the standard deviation increases with the mean value of airflow force. Indeed, from the experimental data obtained by Hall (1988) and his observation that the rms to the mean lift is $\sim 0.2$ for a sphere in a fully developed turbulent boundary layer in an aerodynamically smooth channel (Hall, 1988), Reek and Hall (2001) assumed that the ratio, $\mathrm{f}_{\mathrm{rms}}$, between the arithmetic standard deviation $\sigma$ and the airflow forces average $F_{\text {avg }}, f_{r m s}=\sigma / F_{\text {avg }}$, was 0.2 for the drag forces too. Experimental measurements at the centre axis of the test section of BISE-II wind-tunnel show that this ratio is near 0.05 . Measurements of airflow velocity fluctuations were also achieved near the wall, $1 \mathrm{~mm}$ above the test area with the same technique. Results gave Gaussian distributions for the velocity fluctuations but with higher relative standard deviations than that obtained at the middle of the wind tunnel. Values between 0.10 and 0.30 were obtained at atmospheric pressure for velocity set-points in the range $5-30$ $\mathrm{m} . \mathrm{s}^{-1}$. These values are consistent with the standard value 0.2 , used in the model from Reeks et al. (2001).

Hereafter, some information's are given on surface roughness of the wind tunnel just upstream the test area. If we define a centred plane, parallel to the roughness plane, such that the volumes delimited on both sides of it are equal, $R a$ can be defined as the average roughness corresponding to the average value of the surface relative to the centred plane, i.e.:

$$
R a=\frac{1}{L_{x} L_{y}} \int_{0}^{L_{y}} \int_{0}^{L_{x}} f(x, y) d x d y,
$$

where $L_{x}$ and $L_{y}$ are the surface dimensions and $f(x, y)$ is the surface relative to the centred plan.

The measurement of the relative standard deviations of the velocity fluctuations $1 \mathrm{~mm}$ above the test area was also performed for different surface roughness. For a smooth surface with $R a<0.1 \mu \mathrm{m}$, a beadblasted surface with $R a \approx 1-2 \mu \mathrm{m}$, and a rough surface from gross machining $R a \approx 10 \mu \mathrm{m}$. The results did not show any significant effect of the surface roughness on the relative standard deviations of the velocity fluctuations. We also note that Zhang et al. (2013) studied numerically the influence of nonGaussian airflow forces distribution on particle resuspension using Rayleigh and Johnson SU 
distributions. But these types of distributions are not representative of stationary airflows observed in the BISE-II wind tunnel.

\subsection{Determination of the friction velocity}

In particle resuspension by turbulent airflow, the friction (shear) velocity is a key parameter (see, for example, Henry and Minier, 2014a). The friction velocity in BISE-II, is determined from the pressure drop method which is based on the pressure drop measurement between two points of the wind tunnel. For that purpose, the friction velocity is defined by the relation:

$$
u_{*}^{2}=\frac{1}{2} \mathrm{C}_{\mathrm{f}} \bar{U}^{2},
$$

where $\mathrm{C}_{\mathrm{f}}$ is the friction coefficient, and $\bar{U}$ the mean airflow velocity. The pressure drop, $\Delta P$, between two points at a distance $L$ in the duct and having an equivalent hydraulic diameter $\mathrm{D}_{\mathrm{H}}$, is expressed as:

$$
\Delta P=\wedge \frac{\mathrm{L}}{\mathrm{D}_{\mathrm{H}}} \rho_{f} \frac{\bar{U}^{2}}{2},
$$

where $\Lambda$ is the linear pressure drop coefficient and $\rho_{\mathrm{f}}$ the density of the fluid $\left(\rho_{\mathrm{f}}=1.2 \mathrm{~kg} / \mathrm{m}^{3}\right.$ for air at normal temperature and pressure conditions). Equation (3) can also be written as:

$$
\Lambda=4 \mathrm{C}_{\mathrm{f}} .
$$

Thus, equations (2), (3) and (4) lead to:

$$
u_{*}=\sqrt{\frac{\Delta P \mathrm{D}_{\mathrm{H}}}{4 \rho_{f} \mathrm{~L}}} .
$$

The hydraulic diameter $\mathrm{D}_{\mathrm{H}}$ in BISE-II is $6.66 \mathrm{~cm}$, and the two $\triangle P$ measurement points are set at a distance $L=96 \mathrm{~cm}$. Note that the friction velocity as measured is averaged on the length $\mathrm{L}$. The friction velocities determined experimentally are compared in Figure 2-(c) to the Schlichting (1979) correlation, valid for a turbulent airflow, and given by:

$$
u_{*}^{2}=0.0225 \times U^{7 / 4}\left(\frac{v_{f}}{\mathrm{R}_{\mathrm{H}}}\right)^{1 / 4},
$$

where $v_{\mathrm{f}}$ is the kinematic viscosity of the fluid $\left(\mathrm{m}^{2} \cdot \mathrm{s}^{-1}\right), U$ the free-stream velocity at the centre of the duct $\left(\mathrm{m} . \mathrm{s}^{-1}\right)$ and $R_{H}$ the hydraulic radius $(\mathrm{m})$. The advantage of referring to the Schlichting correlation is to easily account for absolute pressure in the test section through the calculation of the kinematic viscosity according to the air density in the test section. For the range of free-stream velocities studied here, we find a very good agreement between the pressure drop measurements and the Schlichting correlation. The following simplest empirical relationship between the friction velocity and the freestream velocity can also be used:

$$
u_{*}=0.04 \times U+0.076 \text {. }
$$

\section{Characterization of surface and particle deposits}

\subsection{Technique to make particle deposits}

To obtain monolayer deposits of tungsten particles, we used the powder disperser of the Morphologi G3 Malvern optical microscope, whose principle is depicted in figure 3. 

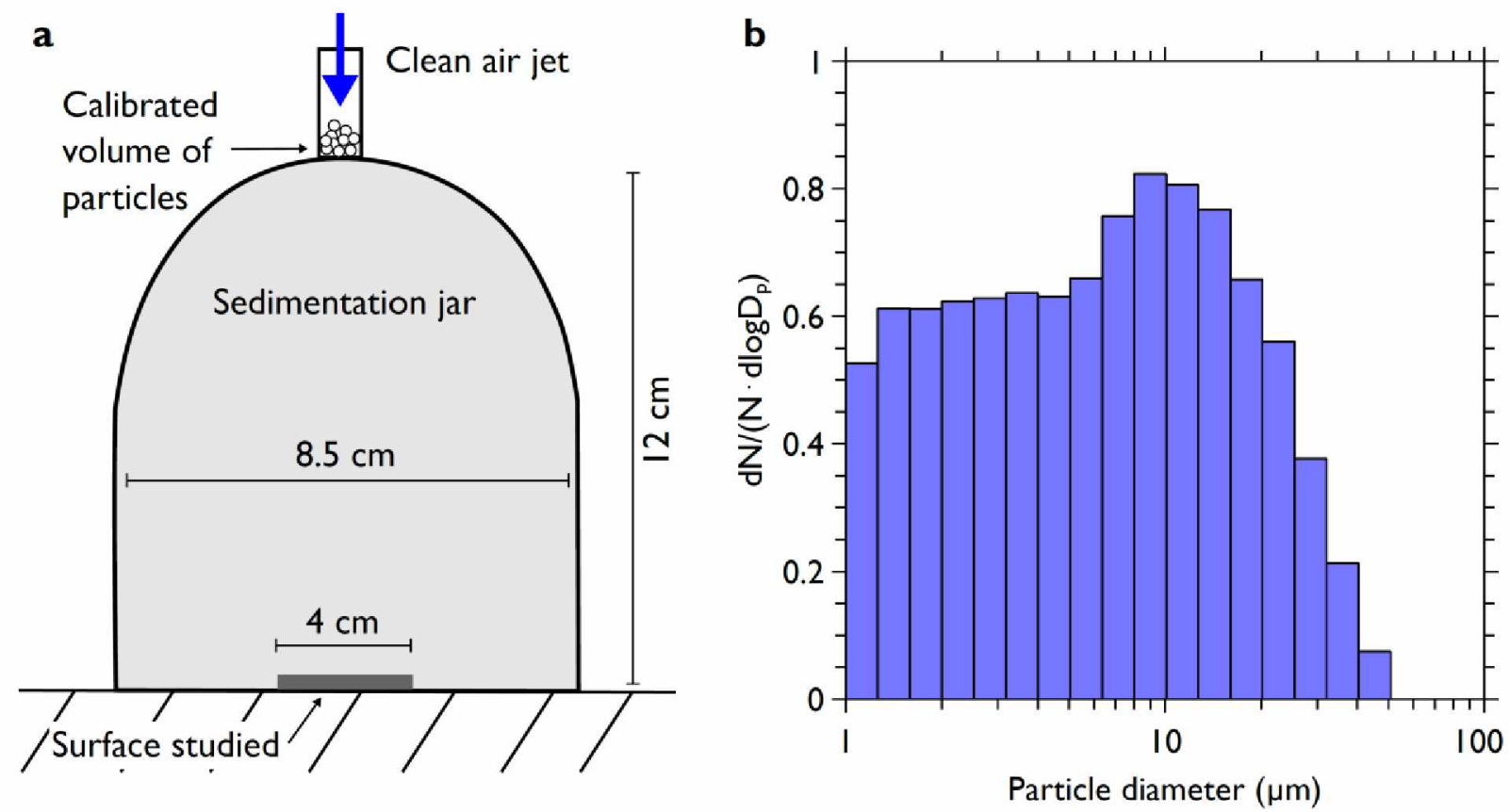

Figure 3-(a) Outline of the device used to produce the monolayer deposits of tungsten particles.

(b) Count size distribution of the particles deposited on the resuspension test area.

This powder disperser is a bell-shaped metallic jar which is set over the surface. The gas tightness is achieved by means of a rubber seal located under the lower edge of the vessel. A calibrated volume (set at 1 or $3 \mathrm{~mm}^{3}$ ) of tungsten particles is poured on the top of the jar. Then, a pulse of clean air, set at an absolute pressure of 4 bars during a time lapse of $10 \mathrm{~ms}$, disperses the tungsten particles inside the jar. The calibrated volume of particles and the air jet were optimised in order to obtain homogeneous monolayer deposits.

To perform resuspension experiments, we used pure tungsten $(>99.9 \%$ ) particles from commercial supplier A.M.L.T. Corp. The count size distribution of particles as deposited on the test surface is measured by the Morphologi $G 3$ microscope. The instrument determines the equivalent diameter of the projected surface area thanks to image processing software. The typical particle size distribution of the deposited particles is displayed in Figure 3-(b). The particles are widely poly-dispersed between $1 \mu \mathrm{m}$ and $50 \mu \mathrm{m}$ with a modal diameter near $8 \mu \mathrm{m}$. Regardless of the specific modal or median diameters, this particle size distribution from A.M.L.T. Corp. was chosen as it covers the micron size range of particles collected in a tokamak with tungsten coating as Plasma Facing Components (PFCs) (see for example Balden et al., 2014, Rondeau et al., 2015b, for the ASDEX tokamak and Peillon et al., 2020 for the WEST tokamak).

For resuspension experiments, the particles are deposited on a rough tungsten surface with a square size of $4 \times 4 \mathrm{~cm}^{2}$. This surface is used since it is a type grade material for plasma facing components in future tokamaks. The surface is oriented such that the roughness lines (see Figure 4) are parallel to the wind flow, thus particles cannot get trapped in the grooves.

Scanning Electron Microscopy (SEM) analysis of particles deposited on the surface is shown in figure 4. One can observe single particles, but also some particle agglomerates. Nevertheless, the individual particles and the agglomerates are well separated and uniformly dispersed on the surface. Indeed, despite the optimization of the powder disperser parameters, we could not completely get rid of particle agglomerates, which is due to the high adhesion forces between tungsten materials and the limited efficiency of the disperser. As the measurements were performed with the optical microscope in the resuspension experiments, agglomerates were considered as single particles having the same equivalent diameter of the projected surface area. The particle surface concentration on the photograph in Figure 4 is equal to $48 \mathrm{~mm}^{-2}$. 


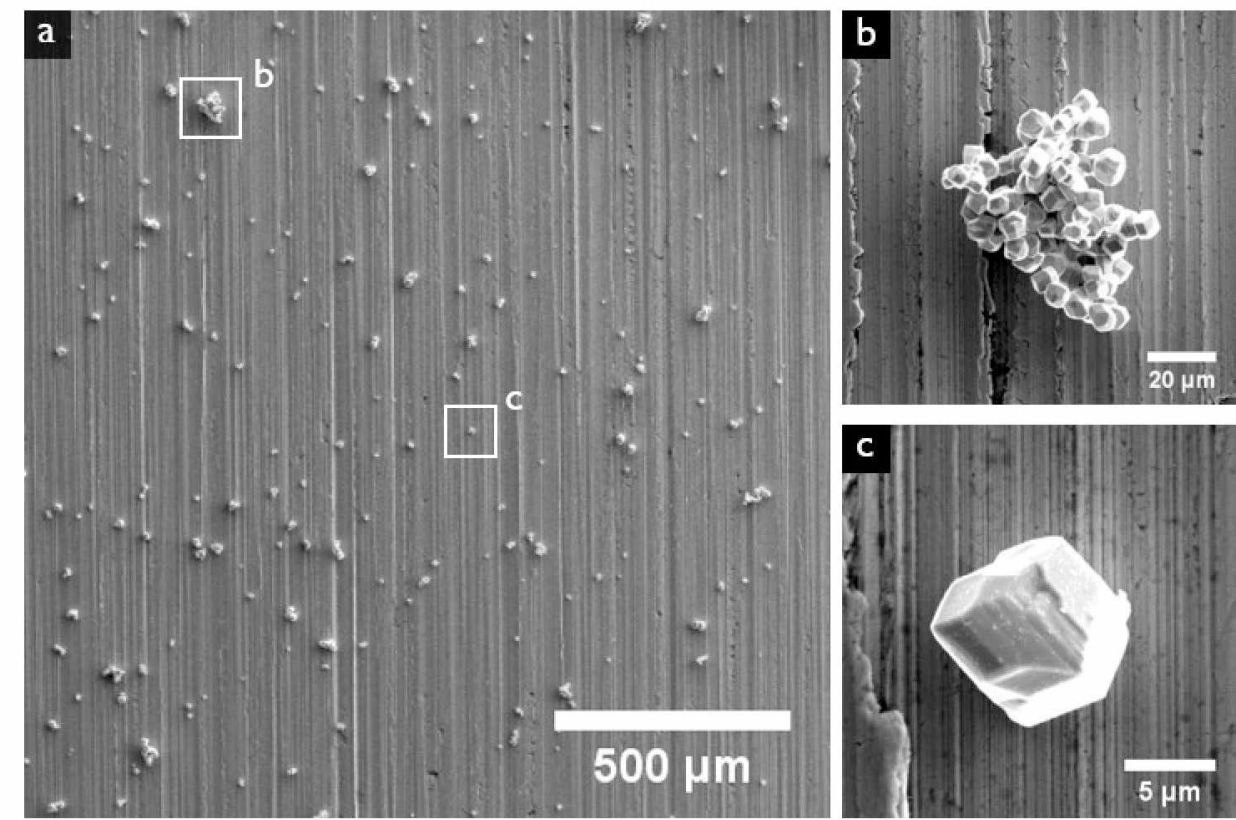

Figure 4-SEM pictures of tungsten particles deposited on a tungsten substrate.

\subsection{Substrate roughness measurement}

Since surface roughness deeply affects the particle adhesion forces (Kumar et al., 2013; Henry and Minier, 2014b; Peillon et al., 2019), the topography of the surface was measured by Atomic Force Microscopy AFM (Scanasyst mode, Bruker Multimode 8) in three areas of $50 \times 50 \mu^{2}$. The average roughness parameter $R a$ (arithmetic mean of all peaks and valleys height) measured was equal to $0.25 \mu \mathrm{m}$, which is a value quite lower than the particle diameters studied. An example of topographic image obtained from an area of the tungsten surface analysis is presented in Figure 5-(a) together with a linear profile extracted from a $50 \mu \mathrm{m}$ length. The average height of the three areas scanned by AFM is depicted by the histogram in Figure 5-b.
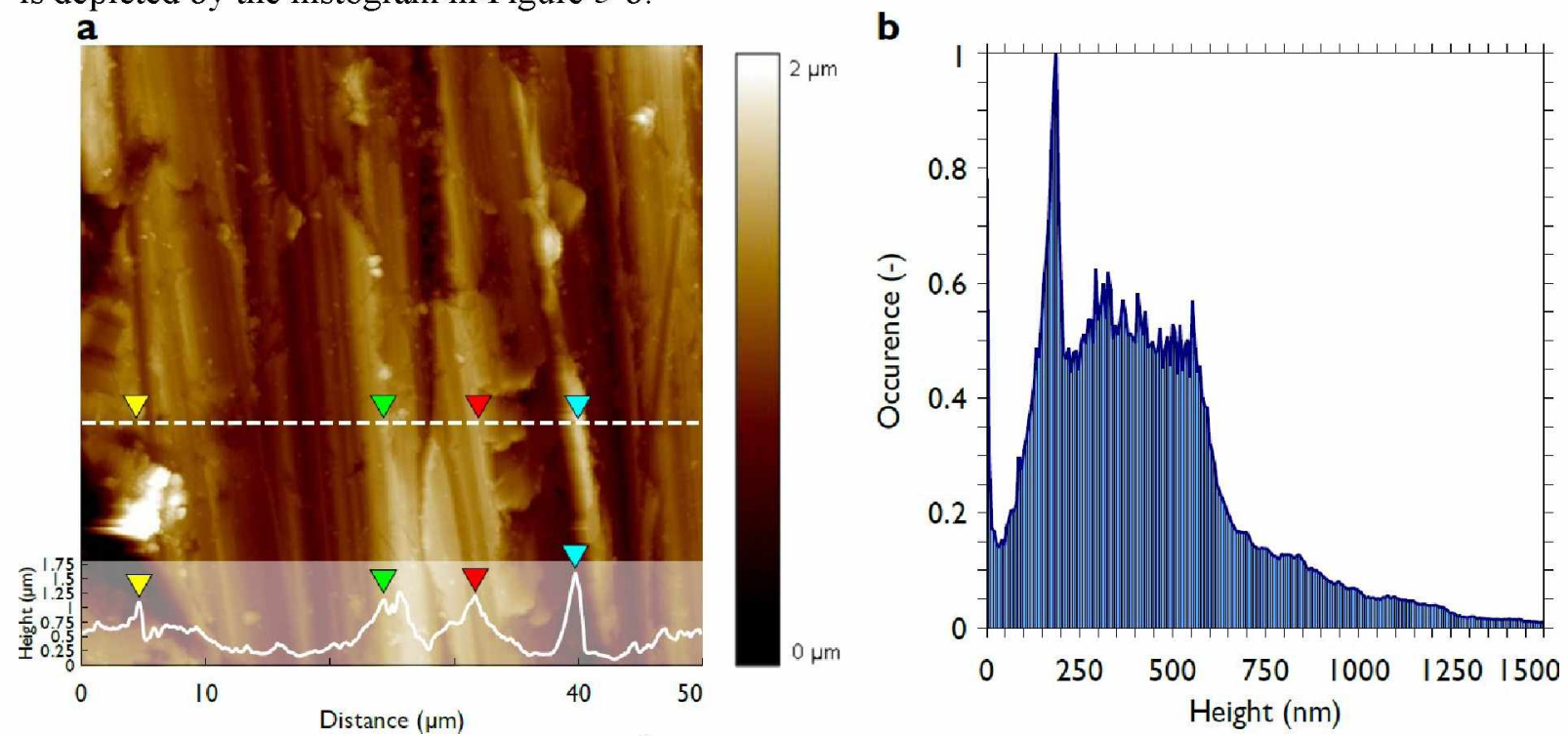

Figure 5- (a) Example of $50 \times 50 \mathrm{um}^{2}$ topographic scan of the tungsten surface with a $z$ scale of $2 \mu \mathrm{m}$. A linear profile is also depicted by the magenta solid line. (b) Histogram of the average height of the surface measured on the three areas. 


\subsection{Adhesion force distribution measurement by Atomic Force Microscopy}

To complete the characterization of particles and deposits regarding resuspension phenomenon, we studied the adhesion force distributions of particles on the substrate used for the resuspension experiments. The measurement was done by the AFM technique (PeakForce Quantitative Nanoscale Mechanical mode) using functionalized cantilevers. Such technique was used in recent experiments of the same kind (Pecault et al., 2012, Mokgalapa et al. 2014, Tan et al. 2014, Feuillebois et al., 2016, Peillon et al. 2019). Five tungsten particles with diameters ranging from $2 \mu \mathrm{m}$ to $24 \mu \mathrm{m}$ were glued onto five cantilevers with stiffness near $2.8 \mathrm{~N} . \mathrm{m}^{-1}$. The particle diameters refer to the equivalent diameter of the projected surface area in a plane parallel to the surface scanned by the AFM. figure 6 shows SEM pictures of the five tungsten particles glued on the AFM cantilevers. Figures 6-(a) to 6-(e) show faces of the particles in contact with the surface. One can notice the shadow of the cantilever behind the particles in Figures 6-(d) and 6-(e).
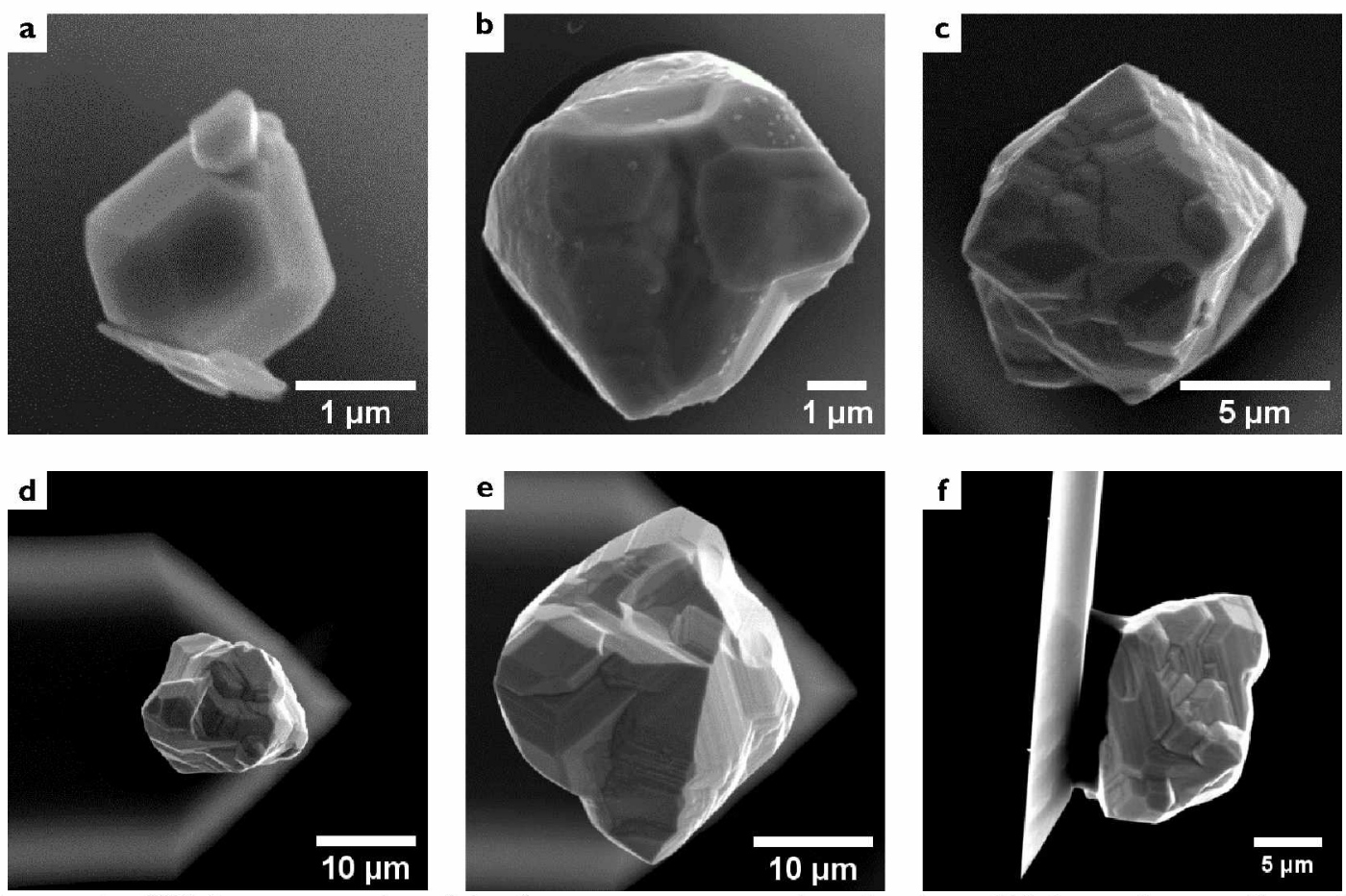

Figure 6-SEM micrographs of the five tungsten particles glued on AFM cantilevers. Particle equivalent diameters are: (a) $2 \mu \mathrm{m}$, (b) $6.3 \mu \mathrm{m}$, (c) $10.6 \mu \mathrm{m}$, (d) $15.5 \mu \mathrm{m}$ and (e) $23.6 \mu \mathrm{m}$.(f) is a side view of the $15.5 \mu \mathrm{m}$ particle presented in $(d)$.

For each functionalized cantilever, five adhesion force measurements were performed on different $50 \times$ $50 \mu \mathrm{m}^{2}$ square areas of the tungsten substrate used for the resuspension experiments. The measurements were carried out in atmospheric conditions at ambient temperature but within a narrow range of relative humidity, namely $38 \%$ to $41 \%$, by selecting the days for measurement according to dry weather conditions to minimize contribution of capillary forces to adhesion. An example of an adhesion force distribution measured on a $50 \times 50 \mu \mathrm{m}^{2}$ square area is given in Figure 7 for a particle with an equivalent diameter of $10.6 \mu \mathrm{m}$. 


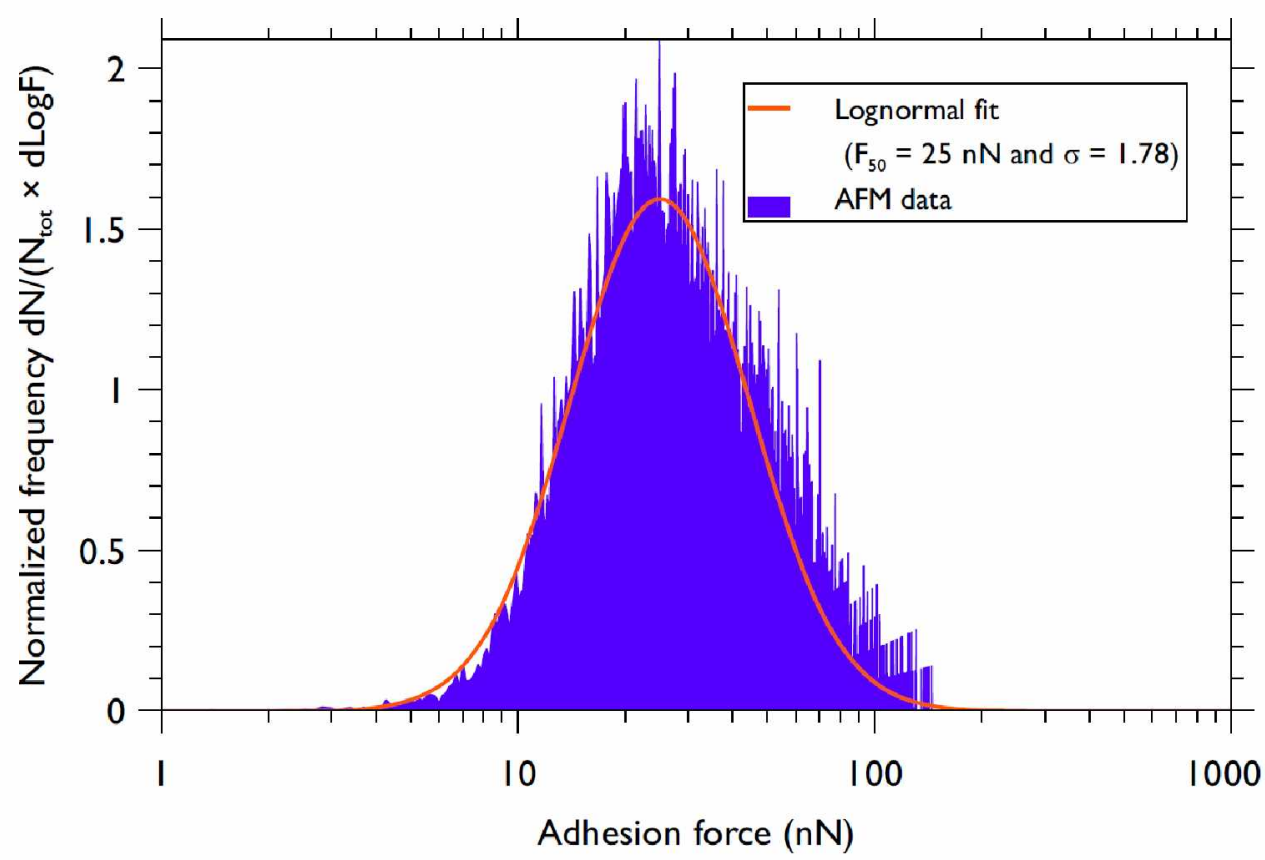

Figure 7 - Experimental AFM measurements for the $10.6 \mu$ m tungsten particle on the tungsten rough surface.

In Figure 7, the median value, $\mathrm{F}_{50}$, of the adhesion force distribution is equal to $25 \mathrm{nN}$. In a recent work, Peillon et al. (2019) measured, with the same AFM technique, a mean adhesion force of $85 \mathrm{nN}$ between a spherical tungsten particle of $11 \mu \mathrm{m}$ in diameter and a tungsten substrate with sub-microscale roughness $(R a=0.12 \mu \mathrm{m})$. The discrepancy between these two values could stem from the difference between surfaces roughness, the rougher it is, the lower the adhesion force. One can understand this effect by the reduction of surface-particle contact area when roughness increases. It should also be noticed that, present results are obtained for a non-spherical particle; consequently a part of the discrepancy with the case studied by Peillon et al. (2019) can also be attributed to particle shape effect on the adhesion. In fact particle shape certainly contributes to variation in surface-particle contact area.

Statistical parameters for each particle studied, namely the median $\mathrm{F}_{50}$ and geometric standard deviation $\sigma$ of the adhesion force distributions obtained from the AFM measurements, are displayed in Figure 8. The reader can access all the measurements made by AFM and lognormal fit of the results in Rondeau (2015).

It can also be noticed in Figure 8 that the mean values of the five median forces determined for each particle increase with particle diameter with an exception for the $23.6 \mu \mathrm{m}$ particle which exhibit a quite low value. This last result may originate from the particular orientation of the particle as it is glued on the cantilever. One can see in Figure 6-(e) that the $23.6 \mu \mathrm{m}$ particle seems to have a peak directly oriented toward the surface scanned during the AFM measurement. This particular result will not be taken into account for the following.

Nevertheless, one should notice that, in the present study, AFM measurements were carried out with non-spherical particles glued on the cantilevers and that only one particle of each size was tested. Consequently, extrapolating these adhesion force distributions for the entire poly-disperse deposit is an important approximation. However, results give first data for irregularly shaped particles and show that the adhesion of non-spherical particles on rough surfaces is an issue for resuspension assessment. 

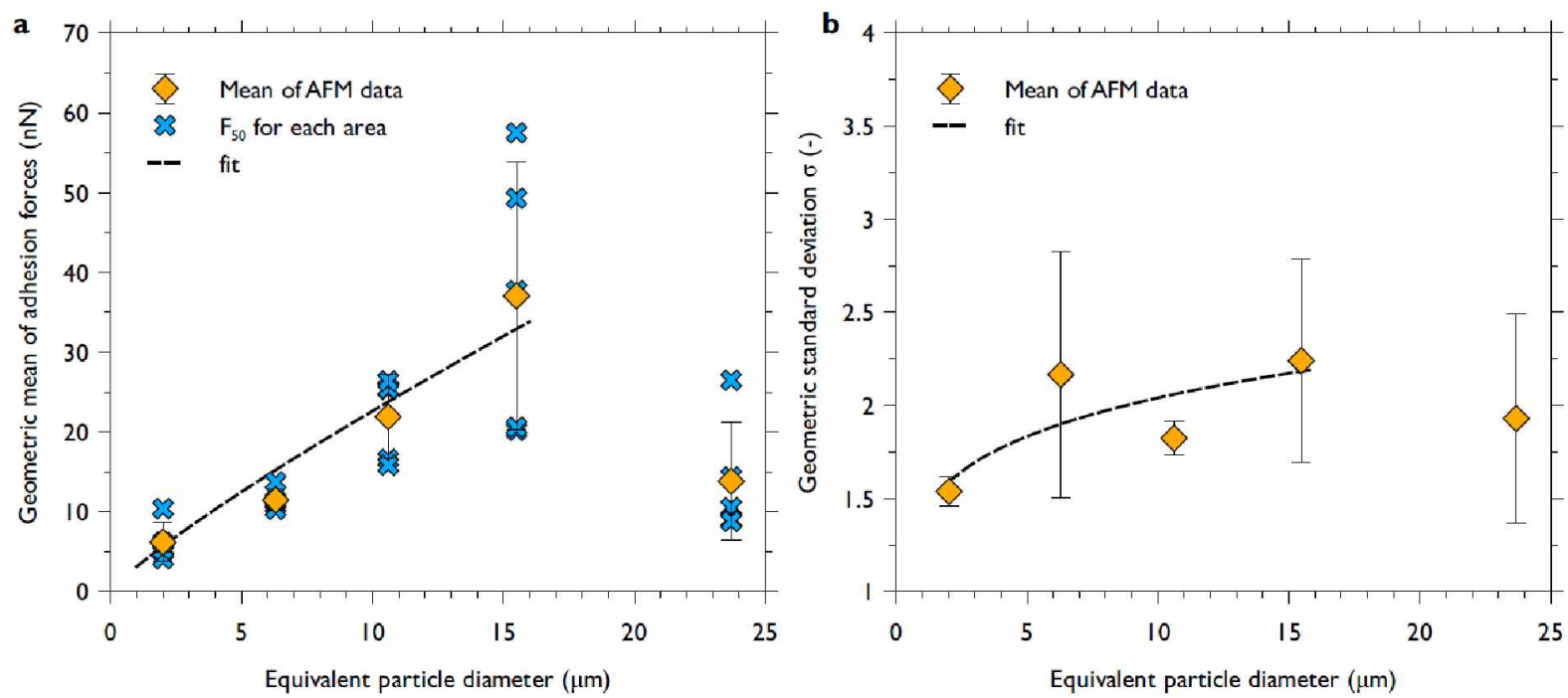

Figure 8-(a) Mean of the five adhesion median force values measured by AFM. (b) Mean of the five geometric standard deviation of force distributions measured by AFM.

For practical use in latter resuspension calculation, the following correlations (represented Fig. 8) for the median adhesion force (in $\mathrm{nN}$ ) and geometric standard deviation are obtained from AFM data for particle diameters up to $16 \mu \mathrm{m}$ :

$$
\mathrm{F}_{50}=3.1542 \mathrm{~d}_{\mathrm{p}}^{0.8558} \text { and } \sigma=1.4295 \mathrm{~d}_{\mathrm{p}}^{0.1546} \text {, }
$$

with $d_{p}$ the particle diameter in micrometre $(\mu \mathrm{m})$. These correlations are represented on Figure 8 together with experimental results.

\section{The resuspension experiments}

\subsection{The measurement technique}

Ibrahim et al. $(2003 ; 2004)$ and Barth et al. (2014) used a lens camera that allowed them to visualize particle diameters above $10 \mu \mathrm{m}$ and to measure the particle resuspension fraction by airflow in a windtunnel. Nevertheless, they could not count and measure enough particles to obtain a classification by size bins with their experimental procedure - image acquisition on line when the particle deposit is exposed to airflow. Thus, they achieved global measurements with monodispersed particle deposits, highlighting the kinetics of the resuspension mechanism.

In the present study, we have performed measurements of particle resuspension from a poly-dispersed deposit by using an optical microscope allowing to scan a large area of the deposit in order to obtain a significant number of particles in several size bins between $1 \mu \mathrm{m}$ and $45 \mu \mathrm{m}$. To do this, we used the Morphologi G3 Malvern instrument. This optical microscope is coupled to a data acquisition system and image processing software. Indeed, with an adequate adjustment (focus, light and threshold), every particle detected is photographed and its size measured. We opted for a compromise between the measurement duration and the counting of a sufficiently high particle number in order to obtain a reliable statistical result. Thus, for each experiment, particle resuspension measurement is performed by scanning the particle deposit on the surface before and after exposure to airflow. The scanning is performed on a circular area $\left(314 \mathrm{~mm}^{2}\right)$ with a diameter of $20 \mathrm{~mm}$, located at the centre of the deposit (tungsten square surface area of $1,521 \mathrm{~mm}^{2}$ ). Since a measurement with the Morphologi G3 lasts eight hours, we set the microscope in a clean laminar airflow hood inside an ISO 7 cleanroom environment to get rid of any contamination by the atmospheric aerosol. The particle resuspension fraction is determined by counting the particle with different sizes on the scanned surface before and after exposure to an airflow lasting ten minutes in the BISE-II wind-tunnel. To obtain enough particles for each size 
bin, we ranked them following a geometrical sequence with a common ratio of 1.26 and an initial value of $1 \mu \mathrm{m}$ (lower sizeable diameter with an acceptable uncertainty). The values presented in the abscissa of the histograms hereafter correspond to the geometric mean of minimum and maximum values of each particle size bin. Thus, using the particle number by size bin, before and after exposure to airflow, we calculated the resuspension fraction, $K r$, of particles for each size bin with the following equation:

$$
K r=1-\frac{N_{f}}{N_{i}}
$$

where $N_{f}$ is the particle number remaining on the substrate after exposure and $N_{i}$ is the particle number initially deposited on the substrate, before exposure to the airflow.

\subsection{Experimental results}

Four sets of resuspension experiments were carried out (see Tab. 1):

- 3 tests at atmospheric pressure (roughly 990 mbar) with a friction velocity of $1.5 \mathrm{~m} . \mathrm{s}^{-1}$;

- 3 tests at atmospheric pressure with a lower friction velocity of $1.0 \mathrm{~m} \cdot \mathrm{s}^{-1}$;

- 3 tests at lower pressure (280 mbar) with a friction velocity of $1.0 \mathrm{~m} \cdot \mathrm{s}^{-1}$;

- 2 tests at atmospheric pressure with a friction velocity of $1.5 \mathrm{~m} \cdot \mathrm{s}^{-1}$ but with reduced particle surface concentration.

The first and second sets were designed to assess the effect of friction velocity, the second and third sets to assess the effect of pressure and the fourth to assess the effect of particle surface concentration. It should be noticed that first, second and third set of experiments were carried out by dispersing $3 \mathrm{~mm}^{3}$ of powder to make the appropriate deposit on the analysed area of $314 \mathrm{~mm}^{2}$. The fourth set was performed with $1 \mathrm{~mm}^{3}$ of powder to decrease particle surface concentration, and the area analysed was increased to $519 \mathrm{~mm}^{2}$ to keep sufficient particle counting in each size bin. In Table 1, experimental values of pressure, friction velocity and mean free-stream velocity in BISE-II are reported. The total particle surface concentration is also reported with two metrics: $C_{\text {surf }}\left(\mathrm{mm}^{-2}\right)$ represents the total particle number concentration and Rs the coverage ratio of the deposit (projected surface of particles divided by the area of the deposit). We also show in Table 1 the minimum and maximum number of particles counted in the whole size bins defined for analysis. The reader can easily access the exact number of particles in each size bin and detailed airflow information in Rondeau (2015) thesis since the test numbering used in table 1 corresponds to the figure numbering of the manuscript where the results are shown.

\section{Table 1 -Parameters of resuspension experiments}

\begin{tabular}{|c|c|c|c|c|c|c|}
\hline Test $^{\circ}$ & $\mathrm{P}(\mathrm{mbar})$ & $\mathrm{u} *\left({\left.\mathrm{~m} . \mathrm{s}^{-1}\right)}^{-1}\right.$ & $\mathrm{U}\left(\mathrm{m} . \mathrm{s}^{-1}\right)$ & $\mathrm{C}_{\text {surf }}\left(\mathrm{mm}^{-2}\right)$ & $\mathrm{Rs}$ & $\mathrm{N}_{\min } ; \mathrm{N}_{\max }$ \\
\hline 5.2 & 989 & 1.5 & 41.0 & 63 & 0.0081 & $147 ; 1620$ \\
\hline 5.3 & 975 & 1.5 & 41.3 & 85 & 0.0130 & $177 ; 2370$ \\
\hline 5.4 & 965 & 1.5 & 41.6 & 47 & 0.0057 & $115 ; 1299$ \\
\hline 5.6 & 971 & 1.0 & 26.8 & 55 & 0.0040 & $34 ; 1626$ \\
\hline 5.7 & 981 & 1.0 & 28.8 & 59 & 0.0047 & $67 ; 1722$ \\
\hline 5.8 & 980 & 1.0 & 27.3 & 53 & 0.0040 & $39 ; 1488$ \\
\hline 5.10 & 281 & 0.9 & 20.6 & 77 & 0.0066 & $30 ; 2226$ \\
\hline 5.11 & 273 & 0.9 & 21.3 & 96 & 0.0088 & $87 ; 3205$ \\
\hline 5.12 & 286 & 1.0 & 25.7 & 47 & 0.0038 & $72 ; 1339$ \\
\hline AR1 & 965 & 1.5 & 41.8 & 36 & 0.0040 & $222 ; 2033$ \\
\hline AR2 & 965 & 1.5 & 41.8 & 40 & 0.0033 & $118 ; 2402$ \\
\hline
\end{tabular}

The results obtained are presented in Figure 9. Figures 9-(a) and 9-(b) show results for tests performed at atmospheric pressure with a friction velocity of $1.5 \mathrm{~m} . \mathrm{s}^{-1}$, and different surface concentrations of the particle deposits. The plots show values of the resuspension fraction lying between 0.4 and 1 , according 
to the particle diameter. The behavior of the smaller particles is especially interesting. For sizes around $4.5 \mu \mathrm{m}$, and below, the resuspended fraction flattens, becoming less dependent on the size. Even more so, for case AR1, a markedly non-monotonous behavior is found. Although we cannot explain the reason for this peculiar finding, it is clear that the surface concentration influences particle resuspension, especially in the case of small particles. Nevertheless, the correlation between surface concentration and resuspension rate is not simple, as will be demonstrated in the next paragraph.

To study the effect of the friction velocity, Figure 9-(c) presents the results obtained at atmospheric pressure with $1.0 \mathrm{~m} \cdot \mathrm{s}^{-1}$. When comparing Figures 9-(a) and 9-(c) it can be noticed that no influence of the friction velocity is conspicuous in the results, i.e., the resuspension fraction values are quite similar in each bin size. Nevertheless, some features are worthy of being remarked at this point. For example, the resuspension rate for the smaller particles is, on average, greater for case 5.7 than for 5.3 but, the concentration is higher for the latter. This apparent contradiction with the results in Figure 9-(b) (where a lower resuspension rate corresponds to a lower concentration value) will be resolved in Section 5, where the hypothesis of collisions is put forth. There, the detachment rate is proposed to depend not on the total concentration of particles but on both the concentration of small and big particles. Up to now, results demonstrate that the total concentration of the particles on the surface has an effect on the resuspension rate for smaller particles

Finally, results shown in Figure 9-(d) highlight the decrease of the resuspension fraction for a reduced pressure. This behaviour shows clearly that the absolute pressure is a key parameter in particle resuspension phenomena. Although lower resuspension fractions were obtained at a lower pressure, the results for particles larger than $4.5 \mu \mathrm{m}$ show the same increasing trend with size as those for atmospheric pressure.

Reduction of the resuspension fraction at low pressure is explained, as a first approximation, as varying according to the aeraulic force exerted on a particle with a defined diameter, proportional to the product $\rho_{\mathrm{f}} \cdot u_{*}^{2}$. As a consequence, at constant friction velocity $u_{*}$, the aeraulic force decreases due to the evolution of the fluid density, $\rho_{\mathrm{f}}$, with pressure. At low pressure (or for submicron particles) one can suspect aeraulic force to weaken due to a slip effect in the transition or the molecular regimes, when the Knudsen number is above 1 . The question is raised by Rondeau et al. (2015a) together with the relevance of the classical Cunningham slip correction factor applied to a particle near or in contact with a surface. The slip effect doesn't seem to prevail in the case studied here, as the variation of fluid density with pressure is much higher than the variation of the Cunningham correction coefficient for pressures and particle sizes at stake in our experiments. A recent progress on this topic has been achieved by $\mathrm{Yu}$ and $\mathrm{Hu}$ (2021) who proposed a slip correction for the drag force in the RnR resuspension model. In fact, for the range of Knudsen numbers $(\mathrm{Kn})$ involved in our experiments realized at $280 \mathrm{mbar}(0.01<\mathrm{Kn}<0.5)$ the Cunningham slip correction factor is close to the slip correction derived by $\mathrm{Yu}$ and $\mathrm{Hu}$ (2021) with discrepancy lower than $20 \%$.

In our experiments, the fluid density varies between $1.17 \mathrm{~kg} \cdot \mathrm{m}^{-3}$ and $0.33 \mathrm{~kg} \cdot \mathrm{m}^{-3}$ for pressures equal to $980 \mathrm{mbar}$ and $280 \mathrm{mbar}$ at $293 \mathrm{~K}$ respectively. Considering a particle size of $5 \mu \mathrm{m}$ in diameter, the classical Cunningham correction coefficient varies between 1.03 and 1.12 respectively for pressures equal to $980 \mathrm{mbar}$ and $280 \mathrm{mbar}$ at $293 \mathrm{~K}$ respectively. The variation is a little more pronounced for the smallest particle diameter studied here $(1 \mu \mathrm{m})$ leading to Cunningham correction coefficients equal to 1.17 and 1.62 for pressures equal to $980 \mathrm{mbar}$ and $280 \mathrm{mbar}$. 

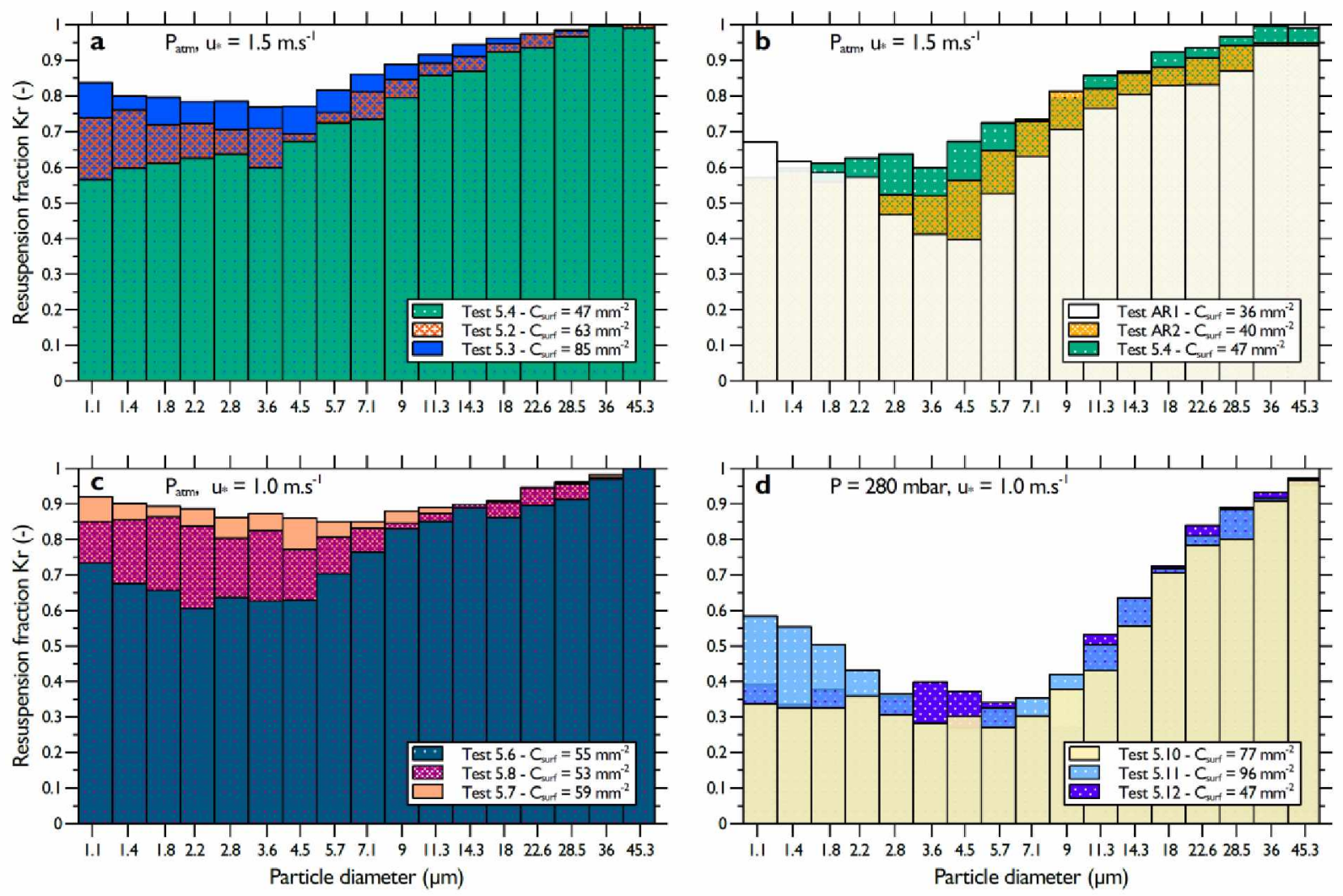

Figure 9 - (a) Resuspension fractions obtained at atmospheric pressure for friction velocity of $1.5 \mathrm{~m} . \mathrm{s}^{-}$

1. (b) Same parameters but with lower particle surface concentrations. (c) Resuspension fractions obtained at atmospheric pressure with friction velocity of $1.0 \mathrm{~m} \cdot \mathrm{s}^{-1}$. (d) Lower absolute pressure of 280 mbar and friction velocity of $1.0 \mathrm{~m} \cdot \mathrm{s}^{-1}$.

\subsection{Comparison with resuspension models}

In order to investigate if the present results can be described by some of the theoretical models on resuspension phenomena, we proceed by averaging the experimental results for the three tests in Figure 9-(a) and those in Figure 9-(c), respectively. Figure 10 presents the mean values obtained for the three tests, error bars representing the standard deviation between resuspension tests.

Results are compared to the calculation done using the RnR model (Reeks and Hall, 2001) and a Monte Carlo (MC) model (Benito et al., 2016). Implementing both models, we followed the main guidelines proposed by the authors in each case, modifying only the values obtained experimentally (friction velocity, adhesion force measurements, particle size distribution and time of exposure to the airflow).

Regarding the RnR model, two versions were tried: one using the correlations from AFM measurements established in section 3 (see eq. 8) and the other using the correlation proposed by Biasi et al. (2001) for the lognormal distribution of adhesion forces. The geometric factor $r / a$ (i.e. the ratio between the particle radius and the adhesion lever arm) is assumed equal to 100 (Reeks and Hall, 2001). For the two versions, we choose the default relative standard deviations for airflow forces equal to 0.2 and both frictions velocities were tested $\left(1.5\right.$ and $\left.1 \mathrm{~m} \cdot \mathrm{s}^{-1}\right)$.

The Biasi et al. (2001) correlation was experimentally established introducing an adhesion force reduction factor which encompasses surface roughness and particle shape effects in comparison to the ideal case described by the Johnson, Kendall and Roberts (JKR) theory (Johnson, Kendall and Roberts, 1971). It is important to notice that JKR theory apply for tungsten-tungsten particle-surface contact deformation in the size range studied here since the Tabor parameter (Tabor, 1977) of the system is 
much greater than 1. The reader could refer to the paper by Autricque et al. (2019) for omnipresence of deformations for particle-surface interactions for tungsten, which is referred as hard metal.

Let discuss the choice of the surface energy of adhesion to be used for calculation with the Biasi correlation. The surface energy of adhesion for W-on-W (tungsten on tungsten) has been derived by Vitos et al. (1998) to be between $4.0-4.6 \mathrm{~J} \cdot \mathrm{m}^{-2}$ depending on the crystal configuration of the surface. Otherwise, Tyson and Miller (1977) carried out surface tension measurements for liquid tungsten and found a surface energy of adhesion of $3.26 \mathrm{~J}^{-2}{ }^{-2}$. One must consider that this surface energy incorporates intrinsically metallic bonding.

Within the JKR theory, the van der Waals surface energy is given by $\gamma=\mathrm{A} /\left(24 \pi \mathrm{z}^{2}\right)$ with $\mathrm{A}$ the Hamaker constant of the system and $z=0.128 \mathrm{~nm}$ the minimal distance between the two surfaces according to Israelachvili (2011). In a recent work, we have measured the Hamaker constant for $\mathrm{W}$-on-W to be $\mathrm{A} \approx$ 3.7.10-19 $\mathrm{J}$ (Peillon et al. 2019). That leads to $\gamma \approx 0.3 \mathrm{~J} \cdot \mathrm{m}^{-2}$ for $\mathrm{W}-\mathrm{W}$ contact at room temperature. The surface energy associated with the metallic bond is thus nearly 10 times larger than the surface energy associated with van der Waals interactions, thus reflecting the relative strengths of the two forces (Tolias et al. 2020).

Moreover, our previous experimental measurements of the pull-off force for tungsten spheres adhered on tungsten smooth surface were roughly one order of magnitude less than JKR contact mechanics predictions and agree within few factors with van der Waals force calculated with the Rabinovitch et al. (2000) model as reported by Peillon et al. (2019). This result has been attributed to the omnipresent of surface roughness. Indeed, as the asperity dimensions begin to exceed the characteristic range of the primary chemical bond, the overall interaction will be progressively dictated by van der Waals forces (Tolias et al. 2020). Hence, even an arithmetic mean roughness $R a$ of few nanometers should suffice to switch the dominant interactions. Thus uncertainty in the value of surface energy of adhesion to be used in the JKR force calculation led us to consider a standard value of $0.56 \mathrm{~J}^{-2}$ used for example by Reeks and Hall (2001) and Ibrahim et al. (2003). The use of this value is also sustained by the fact that Biasi et al. (2001) used this standard value to derive their correlation by fitting experimental data on resuspension when surface energies of particles-surface where unknown. It is also of primary importance to recall that their correlation was established for particle resuspension experiments with diameters between $0.7 \mu \mathrm{m}$ and $30 \mu \mathrm{m}$. Extrapolation out of this range is unphysical as the reduction factor given by the correlation becomes negative for particle diameters above $70 \mu \mathrm{m}$. For this reason, Figure 10 shows dashed lines indicating this issue. In the same way, calculations done with the AFM correlation are stopped at a particle diameter of $16 \mu \mathrm{m}$ according to the limit of the correlation established in Figure 8 .

In the case of the MC model, the resuspension rate is calculated assuming "rolling" as the detachment mechanism governing the phenomenon kinetics, i.e. a detachment based on a moment balance condition. This model was modified in order to take into account the experimental scenario described above: the same initial particle size distribution, a lognormal adhesion force distribution from experiments with AFM measurements, and relative standard deviation of airflow forces equal to 0.2. More details concerning these forces are given in the supplementary materials

Besides, the MC model assumes that the lever arm for the particle detachment condition is constant and equal to $0.5 \mu \mathrm{m}$. This value is of the order of $2 R a$, assuming hemispheric bumps next to each other in agreement with the expected surface topography. At this point, it is important to mention that a prior analysis of the sensitivity of the MC model on the lever arm assumption and on the bursting frequency was done. In first place, results demonstrated that the resuspension rate is severely affected by the leverarm values selected for the model, i.e., 2 Ra instead of the typical assumption of $\frac{r}{a}=100$ made in the RnR model (see Figure 1 of the supplementary materials).

Moreover, we also analysed the adhesion force lever arm effect by extracting this value from a statistical distribution, mimicking a potential spatial distribution of the roughness peak locations. We found that the distribution in the lever arm does not affect significantly the results when compared with the assumption of a constant value. This might simply be due to the fact that those fluctuations will affect the tails of the distance distribution, which are not captured by the global resuspension rate determined by experiments and simulations (see Figures 2 and 3 of the supplementary materials). 
Finally, the effect of the burst frequency is also slight. As a consequence, and in the absence of further information on the surface topography and on the burst kinetics, we used in Figure 10 the parameters mentioned above. The reader will find the sensitivity analysis in the supplementary materials file.

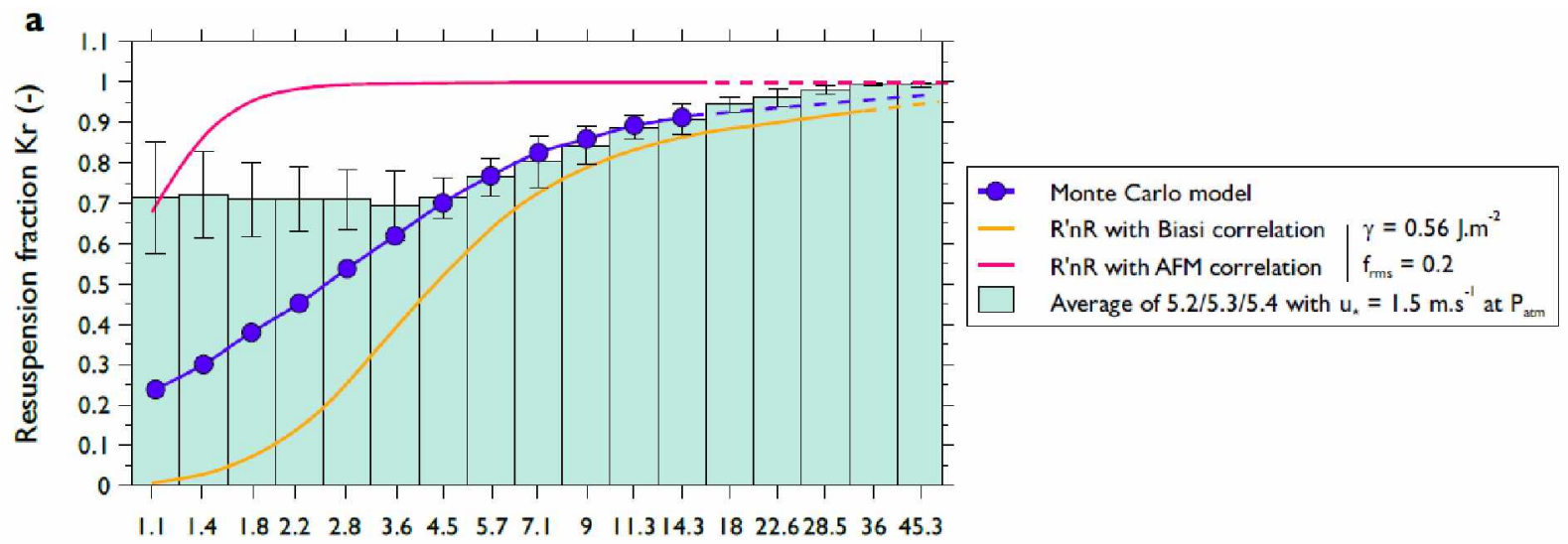

Equivalent particle diameter $(\mu \mathrm{m})$

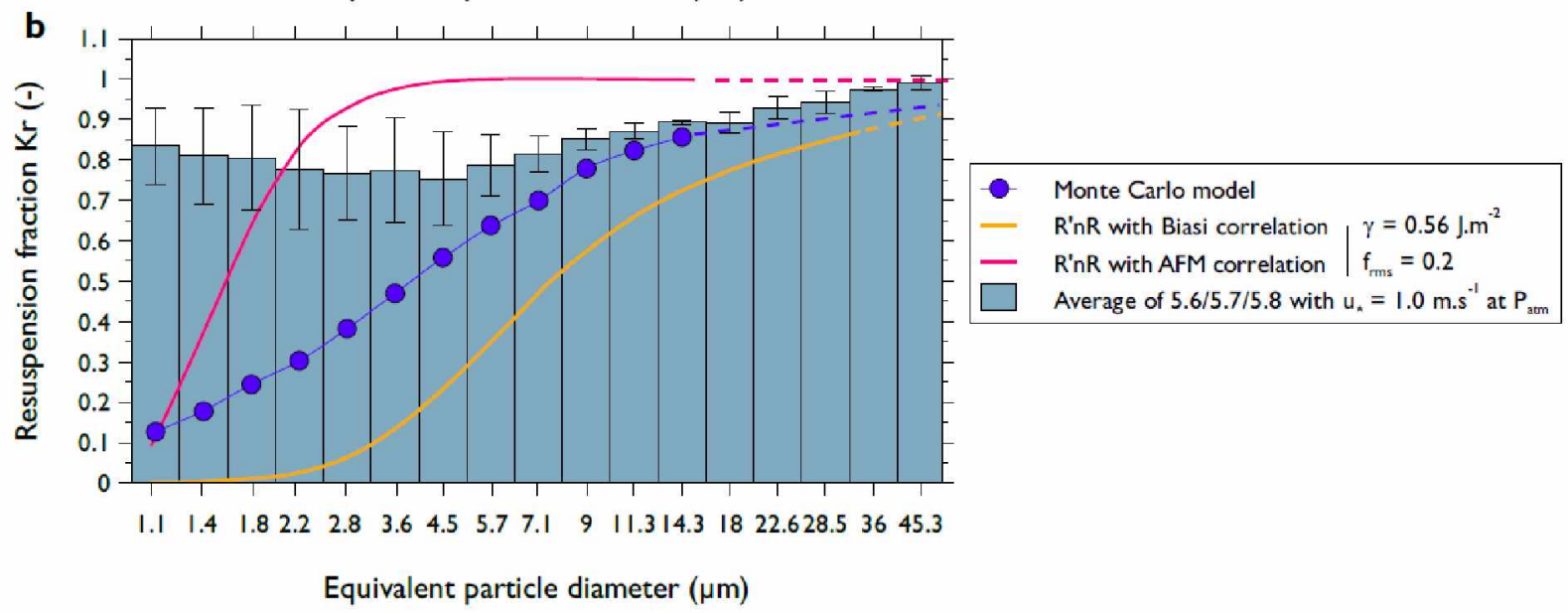

Figure 10 - (a) Comparison between experimental resuspension data of tests 5.2/5.3/5.4 and the different resuspension models. The absolute mean pressure was 976 mbar and the friction velocity $1.5 \mathrm{~m} . \mathrm{s}^{-1}$. (b) Comparison between experimental resuspension data of tests 5.6/5.7/5.8 and the different resuspension models. The absolute mean pressure was 977 mbar and the friction velocity $1.0 \mathrm{m.s} \mathrm{s}^{-1}$.

Figure 10 shows the comparison between the experimental and the numerical results computed from the different tested models. It is clear from the figure that the prediction of the RnR model using the experimental correlation obtained with the AFM data is not consistent, whatever the particle size. The discrepancy may be due to the strong assumption that the relation between the particle radius and the lever arm is constant and equal to 100 . We will return to this point below.

Numerical resuspended fractions predicted by the RnR model using Biasi correlation are more accurate when large particles are at stake. However, the trend shows a clear underestimation of the resuspended fractions, especially for the lower friction velocity.

For the case of the MC model, the predictions are better. The agreement between experimental and numerical results in the range of large particles is very good, showing a satisfactory predictive capacity for our experimental scenarios. The dashed lines represent the values obtained extrapolating the AFM correlation for particle diameter greater than $16 \mu \mathrm{m}$ according to Figure 8. As explained above, other assumptions for the lever arm were tested for the MC model (see supplementary materials). In particular, as pointed out here above, a constant ratio of 100 between the particle radius and the lever arm was implemented. Results were very close to those given by the RnR prediction in Figure 10. For that reason, we think that this assumption should not be used arbitrarily without experimental support (Brambilla and Brown, 2020). 
Although the numerical results are close to the experimental data for particles larger than $4.5 \mu \mathrm{m}$, it is clear that the plateau shown for the resuspension fraction of the smaller particles cannot be assessed through any of the tested configurations.

One should notice that the standard deviation between tests represented in Figure 10 increases significantly as particle size decreases. We check that variability was not due to counting statistics of fine particles. For size bins below $4.5 \mu \mathrm{m}$, the minimum number of particles counted in the area scanned by the microscope is 749 , consequently the expended $(\mathrm{k}=2)$ relative uncertainty on particle counting is $7 \%$. As the counting uncertainty alone could not explain variability of the results, it is thought that an experimental parameter, like particle surface concentration, which is not easy to keep constant, could be involved. Hereafter we assess the effect of particle surface concentration together with collision frequency to explain high resuspension fractions observed for fine particles.

\section{Data analysis according to collision frequency}

Given the limitation of the selected models to explain the high resuspension rate of small particles, the hypothesis of the existence of collisions is worth addressing.

We recall that our experiments do not track the particle kinematics. Consequently, the implementation of dynamical models like the refined version developed by Henry et al., (2012) would be difficult to match with the experimental data without making many assumptions about the way in which nonspherical and poly-dispersed particles move on a rough surface. In addition, the evaluation of the possible impacts between particles should be included in the models.

Therefore, it is important to discuss here some dynamical aspects of the particle-surface coupling underlying our experimental and theoretical approach. In physical and numerical models, it is usually supposed that, once the critical condition for detachment is fulfilled, particles leave the near-wall region and are then transported to the bulk of the flow. If collisions are present, it is necessary that a detached particle remains in the near-wall region at least for a certain time lapse in order to interact with the remaining motionless particles.

In our analysis of collision likelihood, we assume that all the big particles that could detach from the surface have the chance of hitting a small particle. This view could imply several possibilities for the dynamics of the moving particle: $(i)$ to detach and move close to the wall, with a small probability of carrying a small particle with it before leaving the near-wall region; $(i i)$ to roll on the wall and hit a small partner and both resuspend; (iii) to leave the surface alone but then sediment on the surface and eventually collide with a small particle. Any complex combination of the previous three mechanisms may also operate.

Case (ii) has been investigated experimentally by Jiang et al. (2008) and one could eventually be in a position to estimate the velocity of the rolling particle from the values of the involved moments. Nevertheless, in the present scenario, assumptions on the residence time of the particle rolling on the surface are needed. For case (iii), the situation is still more complex to describe.

For these reasons, we propose here a simple statistical estimation of the collision likelihood among particles on the surface by using the experimental concentrations. By a thorough analysis of the experimental data, we correlate this statistical estimation with the experimental resuspension rates.

To evaluate the collision probability, we first assume a "binary" population of particles on the surface: (i) those resuspending only by a rolling mechanism and (ii) those which only resuspend thanks to a collision (or contact) with a rolling particle of the first class. This "binary view" splits the population in "big" and "small" particles, $N_{\text {small }}$ and $N_{\text {big }}$, respectively. Then, a critical diameter $d_{c}$ has to be chosen to separate the whole range of particle sizes into two sets. This value will be determined and discussed later. To ease the calculation, we also assume that collisions occur only between two particles, one big and one small.

With the assumptions above, the respective surface concentrations for the two sets can easily be calculated through: $C_{\text {small }}=\frac{N_{\text {small }}}{\mathrm{S}}$, and $C_{\text {big }}=\frac{N_{\text {big }}}{\mathrm{S}}$, where the denominator $S$ is the corresponding area inspected in the experiments $\left(314 \mathrm{~mm}^{2}\right.$ or $\left.519 \mathrm{~mm}^{2}\right)$.

The resuspended fraction for the small particles, $K_{r s}$, is calculated by taking the average value over the corresponding bins $\left(\leq d_{c}\right)$ in each of the tests performed in Figure 9. For example, for the first test in 
Table 1, when $d_{c}=4.5 \mu \mathrm{m}$ is considered for the splitting, the average value of $K_{r s}$ results is 0.72 , i.e., a value averaging from the 7 bins ranging from 1.1 to $4.5 \mu \mathrm{m}$ (Figure 9).

On the other hand, the mean free path, $\lambda_{m}$, traveled by a big particle with typical size $D_{b i g}$ to encounter a small particle on its way, is:

$$
\lambda_{m} D_{\text {big }} C_{\text {small }}=1 \Rightarrow \lambda_{m}=\frac{1}{D_{\text {big }} C_{\text {small }}},
$$

where $D_{\text {big }}$ is the average size for the big particles calculated from the corresponding particle size distributions of the scanned area in each experiment.

In this way, the collision frequency for a big particle moving with a given velocity $u$ against a small motionless particle is:

$$
v=\frac{u}{\lambda_{m}}
$$

As explained above, we were not able to track the particle's kinematics, that is, to measure its velocity $u$ on the surface. Nevertheless, a conjecture can be made in the absence of any other estimation pertaining to the particle velocity on the surface. It consists in assuming that the velocity on the surface is proportional to the friction velocity $u_{*}$. This is conservative assumption considering that the size range of the particles studied and the friction velocity used ensure that particles are immersed in the viscous sublayer. In addition, it is expected that $v$ will increase with $u_{*}$. For these reasons, we replace $u$ by $u_{*}$ in Equation 11. This conjecture aims to compare the different collision frequencies, despite the unknown actual value of the particle velocity at the wall. Anyway, would this assumption be different, the existence of collisions between particles still requires the drawing up of a correlation like the one derive below.

It is expected that the resuspension rate would be proportional to the collision frequency, where all the small particles are targets shared by all the big particles distributed on the surface. In the present scenario, we assume that once a small particle is collided by a big one, it will detach. Thus, the relative concentration of small to big particles $\left(C_{\text {small }} / C_{\text {big }}\right.$ ) plays a role in the availability of collision events. This means that, depending on the initial set up of particles on the surface, a high concentration of small particles is expected to promote a high resuspension rate and, conversely, a high concentration of big particles will reduce the probability for a big particle to collide with a small particle. Consequently, the expected initial rate of small particles hit by big particles per unit time is given by:

$$
F_{q}=v \cdot \frac{C_{\text {small }}}{C_{\text {big }}}
$$

If collisions are present, $F_{q}$ should account for the effective resuspended fraction of small particles. In other words, although it is not known whether the collided particle finally succeeds to resuspend, a correlation between $F_{q}$ and $K_{r s}$ is a necessary condition for the occurrence of collisions.

It still remains to determine the value for $d_{c}$. To this end, we perform six different trials for $d_{c}: 2.2,3.5$, $4.5,5.6,8.9$ and $14.1 \mu \mathrm{m}$, respectively. As already mentioned, the whole size range of the particles is split into two sets: bigger and smaller than $d_{c}$.

For each trial, we calculate $F_{q}$ following the procedure described by equations (10), (11) and (12), in the case of atmospheric pressure. Figure 11 shows three examples for the correlation between $K_{r s}$ as a function of $F_{q}$, for $d_{c}=3.5,4.5$ and $8.9 \mu \mathrm{m}$, respectively. In each plot, a linear fit quantifies the correlation degree through the coefficient $\mathrm{R}$. It is clear from the figure that the correspondence is not so good for all cases. Indeed, if the R-factors drawn from each of the six fits are plotted as a function of $d_{c}$ (see Figure 12) it turns out that the best correlation is obtained for $d_{c}=4.5 \mu \mathrm{m}(\mathrm{R}=0.96)$. For that reason, the correlation for low pressure is also shown in Figure 11-(b).

The fact that a good correlation is obtained for $d_{c}=4.5 \mu \mathrm{m}$ evidences the presence of collisions in the system and is in agreement with the region where the prediction of the resuspension models depart 
noticeably from the experiments. It is also in correspondence with the relatively more flat-like behavior found for smaller particles, which resuspend quite independently of their sizes.
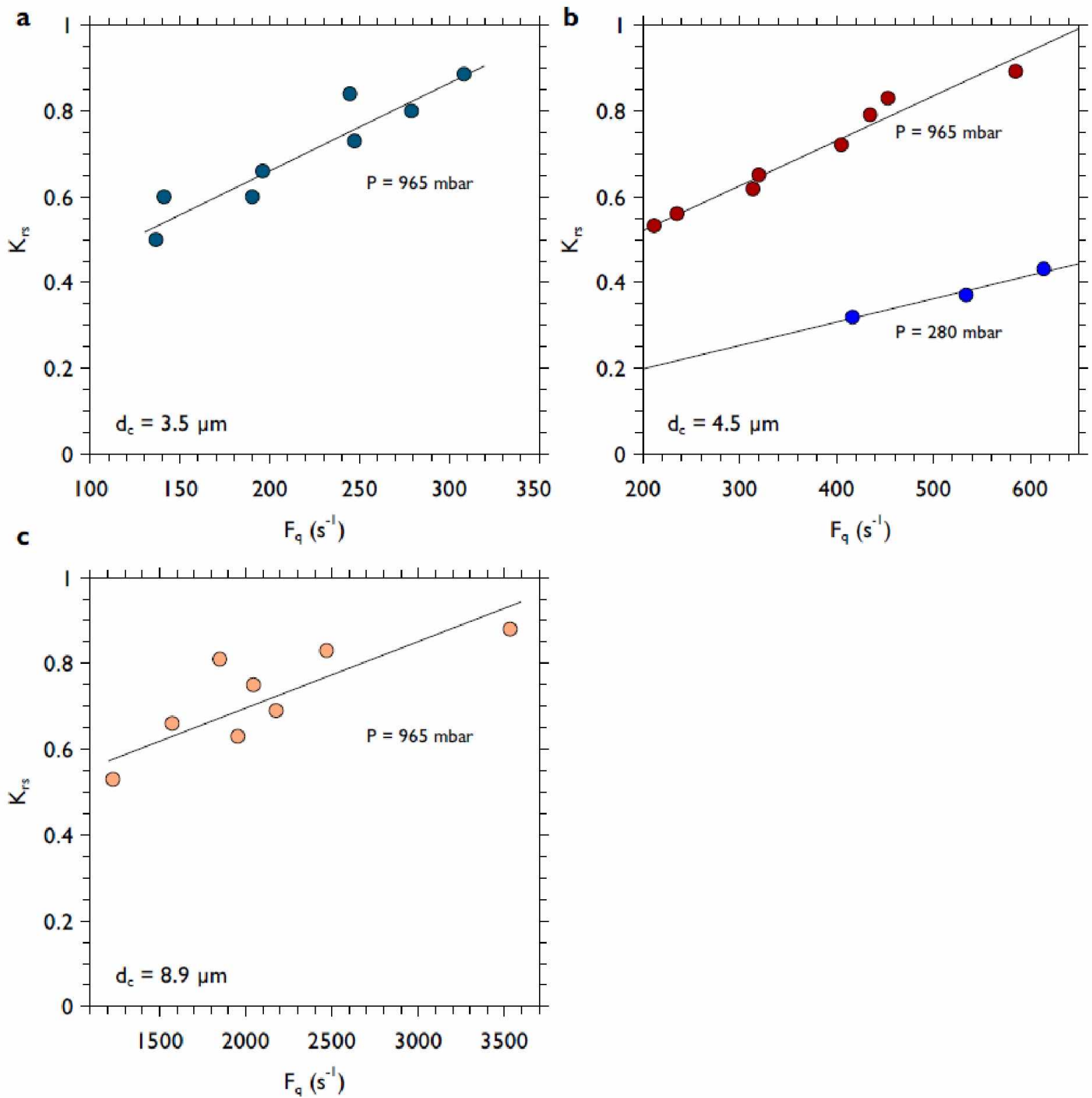

Figure 11 -Linear correlation between the resuspension fraction of small particles, $K_{r s}$, and the collision rate parameter $F_{q}$ for: $(a) d_{c}=3.5 \mu \mathrm{m}$, (b) $d_{c}=4.5 \mu \mathrm{m}$ and (c) $d_{c}=8.9 \mu \mathrm{m}$. The correlation coefficient for the linear fit is $R=0.86,0.96$ and 0.59 , respectively. 


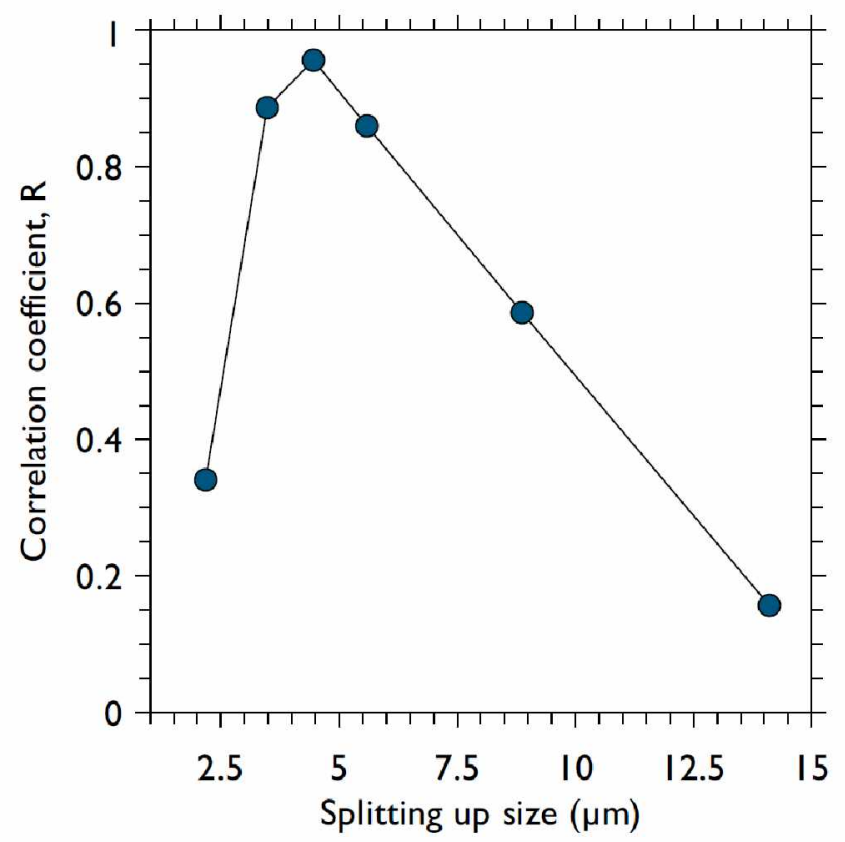

Figure 12 Quality of the different correlations as the size of the splitting up between small and big particles is changed. The maximum value is 0.96 for $4.5 \mu \mathrm{m}$.

\section{Conclusion}

We have performed experiments to study the resuspension of poly-dispersed tungsten particles by a turbulent airflow in a wind tunnel. Experimental conditions were fully characterized, i.e., particle size distributions, particle concentrations, surface roughness, adhesion force distributions and BISE-II wind tunnel airflow conditions. Different tests were conducted to analyze the effect of friction velocity, pressure and particle concentration on the surface.

As a general trend, results show that particle resuspension weakens when the pressure is decreased, while the friction velocities tested in this study did not produce significant differences. Consequently, the influence of the pressure should be thoroughly studied and future efforts are needed in that direction. The recent slip correction derived by $\mathrm{Yu}$ and $\mathrm{Hu}$ (2021) will improve future data analysis and allows to make distinction between fluid density effect and fluid slip effects.

On the other hand, our poly-dispersed system presented an unexpected high resuspension rate for sizes around $4.5 \mu \mathrm{m}$ and below, with the particle concentration clearly affecting the resuspended fractions. The correlation between total surface concentration and resuspension rate is not straight. Depending on the initial set up on the surface, a high concentration of small particles promotes a high resuspension rate and, conversely, a high concentration of big particles reduces the probability for a big particle to hit a small particle. This is a particular feature clearly absent in mono-dispersed or quasi mono-dispersed systems.

When modeling the experiments with different theoretical approaches, a systematic underestimation of the resuspended fraction for small particle sizes $(<4.5 \mu \mathrm{m})$ was found. Only the derivations using RnR model with the AFM correlation highly overestimate the experiments. Moreover, none of the tested models could explain the quasi-plateau observed in the experiments for the small diameters. Nevertheless, model results are consistent with experimental results for the larger particles (again with an exception for the derivations using RnR model and the AFM correlation), MC approach prediction being slightly better when compared with other models.

By assuming a collision effect between particles on the surface, which contributed to resuspension of the smallest particles of the deposit, a good linear correlation was obtained between small particles detachment fraction and a collision parameter, demonstrating that collisions could be responsible for the 
unexpectedly high resuspension rate. Indeed, when particle surface concentrations are high enough (like the ones employed here), one can expect smaller particles to be collided by bigger ones before airflow detachment.

It is important to note that the resuspension dynamics of poly-disperse particles can be very complex, making assumptions necessary in the absence of more detailed information. For instance, clusters of small particles on the surface were considered as a single big particle. These aggregates could also play an important role in the collision mechanism due to clusters higher likelihood of clusters re-deposition. We were not able to address this hypothesis in our experiments and a direct record of the dynamics of the resuspension of poly-dispersed particles is a goal for future work.

In conclusion, particle surface concentration and collision effects are clearly relevant and should be considered in the different theoretical resuspension models when applied to monolayer poly-dispersed particle deposits.

\section{$\underline{\text { Acknowledgements }}$}

We thank Biophy Research, and in particular Chrystelle Reynaud and Simon Desbief, for their state-ofthe-art adhesion force measurements. This work was supported by IRSN (France), CONICET (Argentina) and by the Secretary of Science and Technology of Universidad Nacional de San Luis, Grant P-3-1-0114.

\section{References}

Autricque, A., Gensdarmes, F., Peillon, S., Sow, M. and Grisolia, C. (2019).Adhesion force of W dust on tokamak W plasma-facing surfaces: The importance of the impact velocity. Nuclear Materials and Energy 18 (2019) 345-349.

Alloul-Marmor, L. (2002). Réentrainement par écoulement d'air d'une contamination particulaire déposée sur une surface. Thèse, Université Paris XII.

Balden, M., Endstrasser, N., Humrickhouse, P.W., Rohde, V., Rasinski, M., von Toussaint, U., Elgeti, S., Neu, R., the ASDEX Upgrade Team (2014). Collection strategy, inner morphology, and size distribution of dust particles in ASDEX Upgrade. Nuclear Fusion, 54, Article 073010.

Balme, M. \& Hagermann, A. (2006). Particle lifting at the soil-air interface by atmospheric pressure excursions in dust devils. Geophysical Research Letters, 33, Article L19S01.

Barth, T., P, Preuß, J., Müller, G. and Hampel, U. (2014). Single particle resuspension experiments in turbulent channel flows. Journal of Aerosol Science, 71, 40-51.

Benito J., Uñac R., Vidales A., Ippolito I., (2016). Validation of the Monte Carlo model for resuspension phenomena. Journal of Aerosol Science, 100, 26-37.

Biasi, L., de los Reyes, A., Reeks, M.W. \& de Santi, G.F. (2001). Use of a simple model for the interpretation of experimental data on particle resuspension in turbulent flows. Journal of Aerosol Science, 32, 1175-1200.

Bisquert, D.S., Peñas-Castejón, J.M., \& García-Fernández, G. (2017). The impact of atmospheric dust deposition and trace elements levels on the villages surrounding the former mining areas in a semi-arid environment (SE Spain). Atmospheric Environment, 152, 256-269. 
Boor, B.E., Siegel, J.A., Novoselac A. (2013). Monolayer and multilayer particle deposits on hard surfaces: literature review and implications for particle resuspension in the indoor environment. Aerosol Science and Technology, 355, 417-422.

Brambilla, S. \& Brown, M. (2020). Impact of the adhesion-force lever-arm "a" on the Rock ' $n$ ' Roll resuspension model and how to compute it from contact mechanics. Journal of Aerosol Science 143, 105525

Csavina, J., Field, J., Taylor, M.P., Gao, S., Landázuri, A., Betterton, E.A., and Sáez, A.E. (2012). A review on the importance of metals and metalloids in atmospheric dust and aerosol from mining operations. Science of the Total Environment, 433, 58-73.

Feuillebois, F., Gensdarmes, F., Mana, Z., Ricciardi, L., Monier, C., Le Meur, G., Reynaud, C. \& Rabaud, M. (2016). Three-dimensional motion of particles in a shear flow near a rough wall. Journal of Aerosol Science, 96, 69-95.

Gelain, T., Rondeau, A., Peillon, S., Sabroux, J.C. \& Gensdarmes (2015). CFD modelling of the wall friction velocity field in the ITER tokamak resulting from airflow during a loss of vacuum accident Consequences for particle resuspension. Fusion Engineering and Design, 100, 87-89.

Gradon, L. (2009). Resuspension of particles from surfaces: Technological, environmental and pharmaceutical aspects. Advanced Powder Technology, 20, 17-28.

Hall, D. (1988). Measurements of the mean force on a particle near a boundary in turbulent flow. Journal of Fluid Mechanics, 187, 451-466.

Henry, C. \& Minier, J.-P. (2014a). Progress in particle resuspension from rough surfaces by turbulent flows. Progress in Energy and Combustion Science, 45, 1-53.

Henry, C. \& Minier, J.-P. (2014b). A stochastic approach for the simulation of particle resuspension from rough substrates: Model and numerical implementation. Journal of Aerosol Science, 77, 168-192.

Henry, C., Minier J.P. \& Lefèvre G. (2012) Numerical study on the adhesion and re-entrainment of nondeformable particles on surfaces: the role of surface roughness and electrostatic forces. Langmuir, $28,438-452$

Ibrahim, A.H., Dunn, P.F. \& Brach, R.M. (2003). Microparticle detachment from surface exposed to turbulent air flow: controlled experiments and modelling. Journal of Aerosol Science, 34/6, 765-782.

Ibrahim, A.H., Dunn, P.F. \& Brach, R.M. (2004). Microparticle detachment from surface exposed to turbulent air flow: Effect of flow and particle deposition characteristics. Journal of Aerosol Science, 35/7: 805-821.

Israelachvili, J. N. (2011). Intermolecular and Surface Forces. Academic Press. Elsevier edition.

Jiang Y., Matsusaka S., Masuda H., Qian Y. (2008). Characterizing the effect of substrate surface roughness on particle-wall interaction with the airflow method. Powder Technol., 186, 199-205.

Johnson, K.L., Kendall, K. \& Roberts, A.D. (1971). Surface energy and the contact of elastic solids. Proceedings A, The Royal Society, 324, 301-313.

Kok, J.F., Parteli, E.J.R., Michaels, T.I. é Bou Karam, D. (2012). The physics of wind-blown sand and dust. Reports on Progress in Physics, 75/10(106901). 
Kumar, A and Xin Jiang, T.S. (2013). Role of relative size of asperities and adhering particles on the adhesion force. Journal of Colloid and Interface Science, 409, 211-218.

Mokgalapa, N.M., Ghosh, T.K. \& Loyalka, S.K. (2014). Graphite Particle Adhesion to Hastelloy X: Measurements of the Adhesive Force with an Atomic Force Microscope. Nuclear Technology, 186, 4549.

Moutinho H.R., Jiang C.S., To B., Perkins C., Muller M., Al-Jassim M.M., Simpson L. (2017). Adhesion mechanisms on solar glass: Effects of relative humidity, surface roughness, and particle shape and size, Solar Energy Materials and Solar Cells, 172, 145-153.

Pecault, I., Gensdarmes, F., Basso, G., \& Sommer F. (2012). Performance assessment of probes dedicated to the monitoring of surface particle contamination. Particle and Particle Systems Characterization, 29, 156-166

Peillon, S., Roynette, A., Grisolia, C. \& Gensdarmes, F. (2014). Resuspension of carbon dust collected in Tore Supra and exposed to turbulent airflow: Controlled experiments and comparison with model. Fusion Engineering and Design, 89/11, 2789-2796.

Peillon S., Autricque A., Redolfi M., Stancu C., Gensdarmes F., Grisolia C. \& Pluchery O. (2019). Adhesion of tungsten particles on rough tungsten surfaces using atomic force microscopy, Journal of Aerosol Science, 137, 105431.

Peillon, S., Dougniaux, G., Payet,M., Bernard, E., Pieters, G., Feuillastre, S., Garcia-Argote, S., Gensdarmes, F., Arnas, C., Miserque, F., Herlin-Boime, N., Grisolia, C., Pluchery O. (2020). Dust sampling in WEST and tritium retention in tokamak-relevant tungsten particles. Nuclear Materials and Energy, 24, 100781 .

Rabinovich, Y. I., Adler, J. J., Ata, A., Singh, R. K., \& Moudgil, B. M. (2000a). Adhesion between nanoscale rough surfaces: Measurements and comparison with theory. Journal of Colloid and Interface Science, 232(1), 17-24.

Reeks, M.W. \& Hall, D. (2001). Kinetic models for particle resuspension in turbulent flows: theory and measurement. Journal of Aerosol Science, 32, 1-31.

Renno, N.O. \& Kok, J.F. (2008). Electrical activity and dust lifting on Earth, Mars, and beyond. Space Science Review, 137, 419-434.

Rondeau, A. (2015) Etude de la mise en suspension aéraulique appliquée à la problématique des poussières dans le futur tokamak ITER. Thèse Université Paris-Saclay. Report ISRN/IRSN/2016-189.

Rondeau, A., Merrison, J., Iversen, J.J., A., Peillon, S., Sabroux, J.-C., Lemaitre, P., Gensdarmes, F. and Chassefière, E. (2015a). First experimental results of particle re-suspension in a low pressure wind tunnel applied to the issue of dust in fusion reactors. Fusion Engineering and Design, 98-99, 2210-2213.

Rondeau, A., Peillon, S., Roynette, A., Sabroux, J.-C., Gelain, T., Gensdarmes, F., Rohde, V., Grisolia, C. and Chassefière, E. (2015b). Characterization of dust particles produced in an all tungsten wall tokamak and potentially mobilized by airflow. Journal of Nuclear Materials, 463, 873-876.

Roth, J., Tsitrone, E., Loarte, A., Loarer, T., Counsell, G., Neu, R., Philipps, V., Brezinsek, S., Lehnen, M., Coad, P., Grisolia, C., Schmid, K., Krieger, K., Kallenbach, A., Lipschultz, B., Doerner, R., Causey, R., Alimov, V., Shu, W., Ogorodnikova, O., Kirschner, A., Federici, G. \& Kukushkin, A. (2009). Recent analysis of key plasma wall interactions issues for ITER. Journal of Nuclear Materials, 390, 1-9.

Sclichting, H. (1979). Boundary-Layer Theory. Seventh edition, Mc Graw-Hill, New York. 
Stovern, M., Felix, O., Csavina, J., Rine, K.P., Russell, M.R., Jones, R.M., King, M., Betterton, E.A., and Sáez, A. E. (2014). Simulation of windblown dust transport from a mine tailings impoundment using a computational fluid dynamics model. Aeolian Research, 14:75-83.

Tabor D. (1977) Surface forces and surface interactions. Journal of Colloid and Interface Science, vol. 58,No.1, 2-13.

Tan, C.L.C., Gao, S., Wee, B.S., Asa-Awuku, A. \& Thio B.J.R. (2014) Adhesion of Dust Particles to Common Indoor Surfaces in an Air-Conditioned Environment. Aerosol Science and Technology, 48, 541-551.

Tolias, P., De Angeli, M., Ratynskaia, S., Riva, G., Bassani, P., Ripamonti, D., Nardone, A., Pedroni, M., \& Ricci, D., (2020). Diffusion bonding effects on the adhesion of tungsten dust on tungsten surfaces, Nuclear Materials and Energy, 24, 100765.

Tyson, W. and Miller, W. (1977). Surface free energies of solid metals: Estimation from liquid surface tension measurements. Surface Science, 62(1), 267-276.

Vitos, L., Ruban, A., Skriver, H., and Kollár, J. (1998). The surface energy of metals. Surface Science, 411(1-2), 186-202.

Yu, D and Hu, F. (2021) Application of the "Rock'n'Roll" (RNR) resuspension model with non-zero slip velocities on particle and substrate surfaces. Journal of Aerosol Science, 151, (2021) 105675.

Zhang, F., Reeks, M. \& Kissane, M. (2013). Particle resuspension in turbulent boundary layers and the influence of non-Gaussian removal forces. Journal of Aerosol Science, 58, 103-128.

Zhang T., Yu S., Peng W., Sun Q., Jiang Y., Shi Q. (2017) Resuspension of multilayer graphite dust particles in a high temperature gas cooled reactor. Nuclear Engineering and Design 322, 497-503.

Ziskind, G. (2006). Particle resuspension from surfaces: revisited and re-evaluated. Reviews in Chemical Engineering, 22, 1-123. 